\title{
Interacting dynamic Wannier-Stark ladder driven by a periodic pulse train
}

\author{
Ken-ichi Hino*,† \\ Doctoral Program in Frontier Science, Graduate School of Pure and Applied Sciences, University of Tsukuba, 1-1-1 Tennodai, Tsukuba, \\ Ibaraki 305-8573, Japan
}

Xiao Min Tong* and Nobuyuki Toshima

Doctoral Program in Materials Science, Graduate School of Pure and Applied Sciences, University of Tsukuba, 1-1-1 Tennodai, Tsukuba, Ibaraki 305-8573, Japan

(Received 12 September 2005; revised manuscript received 18 September 2007; published 22 January 2008)

\begin{abstract}
The electronic structures of the Floquet states of the dynamic Wannier-Stark ladder (DWSL) are examined, where the DWSL is formed by driving the biased superlattices (SLs) by the periodic pulse train (PPT) with the electric field $F(t)$-with time $t$ - and the temporal period $2 \pi / \omega$. For a strong $F(t)$, interminiband interactions, namely, the ac-Zener tunneling (ac-ZT), are predominantly caused in the DWSL. Such a system is termed the interacting DWSL. In order to understand the details of the Floquet states and the modulation patterns by alteration of a couple of the PPT laser parameters, the linear absorption spectra, $\alpha_{a b s}\left(\omega_{p} ; \omega\right)$, of optical interband transitions invoked by the monochromatic probe laser $f_{p}(t)$ with the frequency $\omega_{p}$ are calculated, where the spectra are not only linear in $f_{p}(t)$ but also nonlinear in $F(t)$. The exciton effect is not included for the sake of simplicity. For the PPT driving with unit-pulse shapes largely deviated from the square and saw-toothed profiles, the spectra show unexpected dent structures, differing a great deal from the corresponding ac-ZT-free spectra basically similar to those of the original SLs just showing the ascending steplike structure. To deepen the understanding of this anomaly, the spectra of $\alpha_{a b s}^{0}\left(\omega_{p} ; \omega\right) \propto \partial \alpha_{a b s}\left(\omega_{p} ; \omega\right) / \partial \omega_{p}$ are also calculated, whereby the dent structures become spectral dips showing the negative absorption. It is found that such anomalous behavior is attributed to the ac-ZT between different minibands that accompanies emission/absorption of the nonzero net number of photons with $J \omega$ (with $J$ a nonzero integer). This anomaly also shows the unusual time dependence in the dual-time optical susceptibility associated with $\alpha_{a b s}^{0}\left(\omega_{p} ; \omega\right)$. Moreover, the possibility of existence of the negative absorption in the more realistic excitonic spectra is speculated.
\end{abstract}

DOI: $10.1103 /$ PhysRevB.77.045322

PACS number(s): 78.67.Pt, 73.21.- b, 73.40.Gk

\section{INTRODUCTION}

It was demonstrated 35 years ago that the magnetic renormalization of the Landé $g$ factor, $g$, is caused by the application of crossed magnetic fields to the system of atomic hyperfine levels. ${ }^{1}$ This field is composed of the oscillating field $B_{1} \cos (\omega t)$ (with the amplitude $B_{1}$, the frequency $\omega$, and time $t$ ) and the static field $B_{0}$. The resulting effective $g$ factor, $g_{\text {eff }}$, is expressed as $g_{\text {eff }}=(-1)^{N} g J_{N}\left(g \mu_{B} B_{1} / \hbar \omega\right)$ in terms of the $N$ th-order Bessel function of the first kind $J_{N}(\xi)$, where $\hbar$ and $\mu_{B}$ mean the Plank constant divided by $2 \pi$ and the Bohr magneton, respectively, and $N$ is an integer given by the resonant condition $N=g \mu_{B} B_{0} / \hbar \omega$. This enables one to control the hyperfine level structure by tuning $B_{1}$ and $\omega$; especially, $g_{\text {eff }}$ vanishes at zeros of the Bessel function. The system of circular states of a Rydberg atom exposed to the crossed magnetic fields is the analog of this system, providing the possibility of controlling the Zeeman level structure. ${ }^{2}$ The dynamics of the Rydberg atom under a circularly polarized microwave field is another example of this analog, where chaotic motions relevant to the Trojan states are created. ${ }^{3,4}$ On the other hand, the one-dimensional system of a symmetric double quantum well $(\mathrm{QW})$ interacting with an external static electric field and an additional sinusoidal electric field is expressed by the similar Hamiltonian to that of the above-mentioned system of the atomic hyperfine levels. Hence, the resulting effective tunneling splitting between the two wells is determined as well by the Bessel function, with the argument in terms of the corresponding laser parameters. The tunneling probabilities can be coherently suppressed at zeros of this function, which is termed the coherent destruction of tunneling. ${ }^{5,6}$

The similar quantum driven tunneling also occurs in semiconductor superlattices $(\mathrm{SLs})^{7}$ and optical SLs. ${ }^{8}$ The miniband energy dispersion $\epsilon(k)$ of the SLs is approximated by the nearest-neighbor tight-binding (NNTB) model as $\epsilon(k)$ $=\epsilon_{0}-(\Delta / 2) \cos (k d)$, where $\epsilon_{0}, \Delta, k$, and $d$ mean the energy of the miniband center, the miniband width, the crystal momentum, and the spatial period, respectively. It was demonstrated that the wave packet dynamics of the semiconductor SLs driven by the monochromatic electric field, $F(t)$ $=F_{a c} \cos (\omega t)$, with the amplitude $F_{a c}$ and the frequency $\omega$, shows spatial charge localization at zeros of $J_{0}\left(e F_{a c} d / \hbar \omega\right)$, termed dynamic localization (DL), and spatial charge delocalization at other abscissas, termed dynamic delocalization (DDL), ${ }^{9}$ where $F(t)$ is applied along the direction of crystal growth and $e$ means the elementary electric charge. The DL is featured by the collapse of the quasienergy miniband, while the DDL is associated with the finite miniband width. ${ }^{10}$ This intriguing phenomenon is also applicable to biased SLs, termed the Wannier-Stark ladder (WSL), when the resonant condition $N=\Omega_{\text {Bloch }} / \omega$ is satisfied so that the effect of the photon-assisted tunneling is maximized. ${ }^{11}$ Here, the additional static electric field $F_{d c}$ is also applied parallel to $F(t)$,

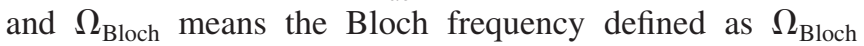
$=e F_{d c} d / \hbar$. The quasienergy of this system is given in terms 


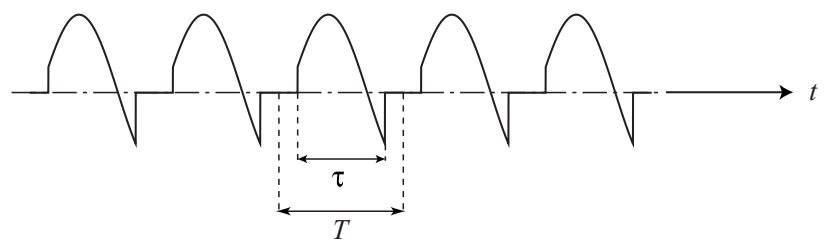

FIG. 1. The illustration of the wave form $F(t)$ as a function of the elapsed time $t$ for the PPT with $x=0.8, \mu=0.7$, and $\delta=\pi / 6$. For $\tau$ and $T$, consult the text.

of $J_{N}\left(e F_{a c} d / \hbar \omega\right)$. Hereafter, a WSL exposed to temporally periodic electric fields is generally termed a dynamic WSL (DWSL); usually, the WSL driven by the monochromatic electric field has been termed the DWSL.

Physics underlying the DWSL is enriched by additional complexity of an excitonic effect, ${ }^{12,13}$ electron correlation, ${ }^{14}$ random disorder, ${ }^{15,16}$ nonlinear coherent dynamics, ${ }^{17,18}$ and interminiband interactions due to the Zener tunneling (ZT). ${ }^{16,19-21}$ Moreover, the DWSL is closely related to diverse phenomena of quantum driven systems, such as the dynamic Franz-Keldysh effect, ${ }^{22} \mathrm{THz}$ radiation, ${ }^{23,24}$ inverse Bloch oscillation, ${ }^{25}$ interacting WSL resonance, and chaotic scattering, ${ }^{26}$ and anomalous effects of coherent timedependent transport. ${ }^{19,20}$ Recently, unusually large induced absorption and electromagnetic transparency were observed below and above the band edge, respectively, in bulk semiconductors strongly driven by intense ultrashort midinfrared laser fields. ${ }^{27,28}$ This is interpreted as a manifestation of the dynamic Franz-Keldysh effect.

Most DWSL systems have been thus far explored in the context of the monochromatic laser driving. Moreover, there are studies of the DWSL arising from a variety of different electric-field wave forms, such as bichromatic waves, ${ }^{13,17,29,30}$ triangular waves, ${ }^{31}$ square waves, ${ }^{32}$ generalized square waves, ${ }^{33}$ and general continuous waves. ${ }^{34}$ Furthermore, the DWSL driven by the time-dependent electric field of the periodic pulse train (PPT) was studied. ${ }^{35}$ This system is examined again in the present paper to obtain deeper understanding. The field of the PPT with the temporal period $T=2 \pi / \omega$ is of the form

$$
F(t)=\sum_{l=-\infty}^{\infty} f(t-l T),
$$

with

$$
f(t)=F_{a c} \cos (\Omega t+\delta) \Theta(t+\tau / 2) \Theta(-t+\tau / 2),
$$

where $\Theta( \pm t+\tau / 2)$ is the Heaviside function, and each pulse unit has the sinusoidal profile with the amplitude $F_{a c}$, the frequency $\Omega$, the constant built-in phase $\delta(0 \leqslant \delta \leqslant \pi)$, and the pulse width $\tau(\tau \leqslant T)$. The pulse given by Eqs. (1) and (2) is featured by a set of the parameters $x=\Omega / \omega, \mu=\tau / T$, and $\delta$. Figure 1 illustrates the wave form of the PPT, for instance, for $x=0.8, \mu=0.7$, and $\delta=\pi / 6$. The PPT under consideration becomes a $\delta$-pulse train for $\mu \ll 1$, a monochromatic electric field for $x=\mu=1$, and a half-cycle pulse train for $x=1$ and $\mu=0.5$.
It is worth mentioning the current status of technique for generating a short pulse train with high repetition rate, which is closely related to the PPT concerned here. The optical Fourier synthesizer was proposed more than one decade ago for generating subfemtosecond light pulses, triangular- and rectangular-shaped pulse trains, and so on by superposition of precisely equidistant optical frequencies, namely, the optical comb, using separate phase-locked laser oscillators. ${ }^{36}$ It was reported that based on this proposal, such a pulse train was actually generated by virtue of some techniques facilitating the Fourier synthesis among subharmonic pulses, for instance, the technique for control of relative carrierenvelop-offset phases among the subharmonic pulses by means of passive pulse-timing synchronized systems. ${ }^{37,38}$ Further, it was recently reported that ultrashort pulse trains were successfully produced through Fourier syntheses of vibrational-Raman sidebands in deuterium ${ }^{39,40}$ and phasecoherent rotational-Raman sidebands in parahydrogen. ${ }^{41}$ The above-mentioned studies are important steps toward establishing the optical Fourier synthesis and the Raman-sideband synthesis of an ultrashort pulse train as an actual light source in the future. It is the coherent control of light-matter interactions that is expected as one of the potential applications of this novel light source.

The primary purpose of this paper is related to the coherent control of the interacting DWSL (IDWSL) driven by the PPT by tuning a variety of the laser parameters. Hereafter, the IDWSL is meant by the DWSL incorporating interminiband interactions due to the dynamic ZT (ac-ZT). The modulation of such interminiband couplings in terms of the laser parameters is particularly focused here. In a previous paper, ${ }^{35}$ the quasienergy of the Floquet state of the IDWSL was studied, and it was shown that the interminiband couplings can be coherently removed by the pulse train with either the square-wave form $(x \ll 1, \mu=0.5, \delta=\pi)$ or the saw-toothed wave form $(x \ll 1, \mu=1, \delta=\pi / 2)$. Usually, the static ZT (dcZT) and ac-ZT are considered as inevitable effects when an applied electric field is sufficiently large. Indeed, knowing the alteration patterns of the quasienergies is the first step necessary to obtain the overall characterization of the quantum driven system concerned. However, aside from such a novel effect of the coherent removal of the ZTs, just an exploration of the quasienergy is not enough to deeply understand the "electronic structure" of the IDWSL and the "modulation pattern" generated by tuning the PPT parameters. In fact, a sequence of the photon sidebands of the quasienergy as a function of $e F_{a c} d / \hbar \Omega$ forms a repeated congruent figure; therefore, without knowing the details of the wave functions, it seems impossible to clarify the difference of the electronic structures between these sidebands.

For the purpose of studying the electronic structures of the IDWSL, here, optical interband transitions between the electron and hole Floquet states of the IDWSL are taken into account, where the transitions are invoked by the monochromatic probe laser with the electric field $f_{p}(t)=f_{p 0} \cos \left(\omega_{p} t\right)$, where $f_{p 0}$ and $\omega_{p}$ represent the amplitude and the frequency, respectively. The resulting effective absorption spectra 
$\alpha_{a b s}\left(\omega_{p} ; \omega\right)$ with respect to the probe laser are not only linear in $f_{p}(t)$ but also nonlinear in $F(t)$. The primary findings are as follows. For the pulse train with the saw-toothed wave form, as is expected from the coherent removal of the ZT in the quasienergy, the spectra show the sequence of the ascending steplike profile that is the same as those of the original SLs; this type of profile is reminiscent of the quasi-twodimesional density of state. On the other hand, for the PPTs that deviated from this wave form by changing $x$ with keeping $\mu$ and $\delta$ unchanged, the spectra show an unexpected dent structure. Almost the same results are also applied to the case of the square-wave form. This anomaly, which is exclusively due to the ac-ZT, is the intriguing issue that has been unexplored thus far. For more expanded analyses, the spectra, $\alpha_{a b s}^{0}\left(\omega_{p} ; \omega\right) \propto \partial \alpha_{a b s}\left(\omega_{p} ; \omega\right) / \partial \omega_{p}$, are introduced, in which the above-mentioned dent structure leads to the negative absorption. The origin of this anomalous negative absorption will be discussed later in further detail.

The quasienergy and the associated effective absorption spectra of the IDWSL driven by the PPT are formulated in Sec. II. To begin with, in Sec. II A, the macroscopic polarization of the present system is derived based on the semiconductor Bloch equations. The equations are solved by employing the Floquet state wave functions of the IDWSL as an expansion basis set. The method of calculating a set of these wave functions is presented in Sec. II B. Finally, in Sec. II C, the expression of the effective absorption coefficient is provided by using the results of Secs. II A and II B. The results and discussion are given in Sec. III, where Secs. III A-III E present the quasienergies, the effective absorption coefficients of $\alpha_{a b s}\left(\omega_{p} ; \omega\right)$ and $\alpha_{a b s}^{0}\left(\omega_{p} ; \omega\right)$, the exploration of the origin of the anomaly, and the miscellaneous issues in a separate manner. Section IV presents the summary and conclusion. Moreover, Appendixes A and B are included. In Appendix A, the approximations and assumptions made in this paper are enumerated for the aid of presentation; further, the criteria of validity for some crucial approximations are shown. In Appendix B, the dual-time optical susceptibility is introduced to supplement the discussion made in the text. The a.u. is used throughout this paper unless otherwise stated.

\section{FORMULATION}

\section{A. Macroscopic polarization}

The macroscopic polarization $P(t)$ for the interband optical transition between the electron and hole Floquet states of the IDWSL is formulated based on the semiconductor Bloch equations. ${ }^{42}$ Here, this polarization is linear with respect to $f_{p}(t)$, while the contribution of the coupling field $F_{T}(t)$, where $F_{T}(t)=F_{d c}+F(t)$, is incorporated into it to the infinite order. In this sense, the spectra obtained from the macroscopic polarization are regarded as the effective absorption spectra. Here, $F_{T}(t)$ is applied along the direction of crystal growth of the SLs, and $F_{d c}$ represents the static electric field, giving rise to the WSL. In the present formulation are made the approximations where only a single electron-hole pair is considered and the Coulomb interactions for the exciton ef- fect and the electron correlation effect are neglected since the present study is aimed at the first characterization, rather than the full analysis, of the IDWSL spectra that have remained unexplored thus far. However, the speculation of the exciton effect on the spectra will be made in Secs. III B and III C

The total Hamiltonian under consideration is given as

$$
\hat{H}=\hat{H}_{0}+\hat{H}^{\prime}+\hat{v}
$$

where $\hat{H}_{0}, \hat{H}^{\prime}$, and $\hat{v}$ represent the joint-miniband Hamiltonian composed of the conduction $(c)$ band and valence $(v)$ band field-free Hamiltonians, the intersubband interaction driven by $F_{T}(t)$, and the interband interaction invoked by $f_{p}(t)$, respectively. Because of $F_{a c} \gg f_{p 0}$ and $\omega \ll \omega_{p}$, it is assumed that $F(t)$ does not contribute to the interband transitions and $f_{p}(t)$ does not contribute to the intersubband transitions. Further, the NNTB model is employed for $\hat{H}_{0}$, the criterion of the validity for which is discussed in Appendix A. Thus, the operators of Eq. (3) are cast into the forms

$$
\begin{gathered}
\hat{H}_{0}=\sum_{s, \lambda, \mathbf{K}_{\|}}\left[(-1)^{b+\sigma^{(s)}} \frac{\Delta_{b}^{(s)}}{4}\left(\hat{\rho}_{\lambda \lambda_{(+1)}(s s)}^{(s)}+\hat{\mathbf{\rho}}_{\lambda_{(+1)}}^{(s s)} \mathbf{K}_{\|}\right)\right. \\
\left.+\left(\epsilon_{\|}^{(s)}\left(\mathbf{K}_{\|}\right)+\epsilon_{0 b}^{(s)}\right) \hat{\rho}_{\lambda \lambda \mathbf{K}_{\|}}^{(s s)}\right] \\
\hat{H}^{\prime}=-F_{T}(t) \sum_{s, \lambda, \lambda^{\prime}, \mathbf{K}_{\|}} Z_{\lambda \lambda^{\prime}}^{(s)} \hat{\rho}_{\lambda \lambda^{\prime} \mathbf{K}_{\|}}^{(s s)},
\end{gathered}
$$

and

$$
\hat{v}=-f_{p}(t) \sum_{\lambda, \lambda^{\prime}, \mathbf{K}_{\|}}\left[d_{\lambda \lambda^{\prime}}^{(c v)} \hat{\rho}_{\lambda \lambda^{\prime} \mathbf{K}_{\|}}^{(c v)}+(\text { H.c. })\right],
$$

with $s$ being the band index $[s=c, v]$, and $\sigma^{(c)}=0$ and $\sigma^{(v)}$ $=1 . \lambda^{(\prime)}$ represents the lump of the miniband index $b^{(1)}$ and the lattice site $l^{(\prime)}$, namely, $\lambda^{(\prime)}=\left(b^{(\prime)}, l^{(\prime)}\right)$, and $\lambda_{(\Delta l)} \equiv(b, l$ $+\Delta l$ ), where $\lambda_{(0)}$ is simply expressed as $\lambda$ for the sake of simplicity, namely, $\lambda_{(0)} \equiv \lambda$. In addition, $\mathbf{K}_{\|}$is the in-plane momentum of a pair of the electrons of the $c$ and $v$ bands, where it is associated with the relative motion of these two electrons in the plane normal to the direction of crystal growth. Further, $\hat{\rho}_{\lambda \lambda^{\prime} \mathbf{K}_{\|}}^{\left.(s)^{\prime}\right)}$ is the density matrix operator given by

$$
\hat{\rho}_{\lambda \lambda^{\prime} \mathbf{K}_{\|}}^{\left(s s^{\prime}\right)}=a_{\lambda \mathbf{K}_{\|}}^{(s) \dagger} a_{\lambda^{\prime} \mathbf{K}_{\|}}^{\left(s^{\prime}\right)},
$$

where $a_{\lambda \mathbf{K}_{\|}}^{(s) \dagger}\left(a_{\lambda \mathbf{K}_{\|}}^{(s)}\right)$ represents the creation (annihilation) operator of the electron with $\lambda$ and $\mathbf{K}_{\|}$in band $s$, and this satisfies the usual anticommutation relation. In Eq. (4), $\Delta_{b}^{(s)}$ is the width of miniband $b$ in band $s, \epsilon_{0 b}^{(c / v)}$ is the center of miniband $b$ reckoned from the bottom/top of the $c / v$ band, $\epsilon_{\|}^{(c)}\left(\mathbf{K}_{\|}\right)=\mathbf{K}_{\|}^{2} / 2 m_{\|}^{(c)}+E_{g}$, and $\epsilon_{\|}^{(v)}\left(\mathbf{K}_{\|}\right)=-\mathbf{K}_{\|}^{2} / 2 m_{\|}^{(v)}$, with $m_{\|}^{(s)}$ being the in-plane effective mass in band $s$ and $E_{g}$ being the band gap between the bands of $c$ and $v$ at the $\Gamma$ point. In Eq. (5), $Z_{\lambda \lambda}^{(s)}$ represents the dipole interaction between the sites of $\lambda$ and $\lambda^{\prime}$ in band $s$ with $Z_{\lambda \lambda^{\prime}}^{(s)}=Z_{\lambda^{\prime} \lambda}^{(s)^{*}}$. In Eq. (6), $d_{\lambda \lambda^{\prime}}^{(c v)}$ represents the dipole interaction between the sites of $\lambda$ in band $c$ 
and of $\lambda^{\prime}$ in band $v$ with $d_{\lambda \lambda^{\prime}}^{(c v)}=d_{\lambda^{\prime} \lambda}^{(v c)^{*}}$, and (H.c.) is meant by taking the Hermitian conjugate of the immediately front term. It is assumed that the dipole interactions given in both Eqs. (5) and (6) are independent of $\mathbf{K}_{\|}$.

The Liouvílle equation

$$
i\left(\frac{d}{d t}+\frac{1}{T_{2}}\right) \hat{\rho}_{\lambda \lambda^{\prime} \mathbf{K}_{\|}}^{(v c)}=\left[\hat{\rho}_{\lambda \lambda^{\prime} \mathbf{K}_{\|}}^{(v c)}, H\right]
$$

yields the following equation for the microscopic polarization of the interband transition $p_{\lambda \lambda^{\prime} \mathbf{K}_{\|}}(t)$ :

$$
\begin{aligned}
i\left(\frac{d}{d t}+\frac{1}{T_{2}}\right) p_{\lambda \lambda^{\prime} \mathbf{K}_{\|}}(t)= & (-1)^{b^{\prime}} \frac{\Delta_{b^{\prime}}^{(c)}}{4}\left[p_{\lambda \lambda_{(+1)}^{\prime} \mathbf{K}_{\|}}(t)+p_{\lambda \lambda_{(-1)}^{\prime} \mathbf{K}_{\|}}(t)\right]+(-1)^{b} \frac{\Delta_{b}^{(v)}}{4}\left[p_{\lambda_{(+1)}}{ }^{\prime} \mathbf{K}_{\|}(t)+p_{\lambda_{(-1)} \lambda^{\prime} \mathbf{K}_{\|}}(t)\right]+\left[\left(\epsilon_{\|}^{(c)}\left(\mathbf{K}_{\|}\right)+\epsilon_{0 b^{\prime}}^{(c)}\right)-\left(\epsilon_{\|}^{(v)}\right.\right. \\
& \left.\left.\times\left(\mathbf{K}_{\|}\right)+\epsilon_{0 b}^{(v)}\right)\right] p_{\lambda \lambda^{\prime} \mathbf{K}_{\|}}(t)-F_{T}(t) \sum_{\eta}\left[Z_{\lambda^{\prime} \eta}^{(c)} p_{\lambda \eta \mathbf{K}_{\|}}(t)-Z_{\lambda \eta}^{(v)} p_{\eta \lambda^{\prime} \mathbf{K}_{\|}}(t)\right]-f_{p}(t) \sum_{\eta}\left[d_{\lambda^{\prime} \eta^{\prime}}^{(c v)} n_{\lambda \eta \mathbf{K}_{\|}}^{(v)}(t)-d_{\eta \lambda}^{(c v)} n_{\eta \lambda^{\prime} \mathbf{K}_{\|}}^{(c)}(t)\right],
\end{aligned}
$$

where the phenomenological dephasing time $T_{2}=1 / \gamma$ has been introduced. Further, $p_{\lambda \lambda^{\prime} \mathbf{K}_{\|}}(t)=\left\langle\hat{\rho}_{\lambda \lambda^{\prime} \mathbf{K}_{\|}}^{(v c)}\right\rangle$ and $n_{\lambda \lambda^{\prime} \mathbf{K}_{\|}}^{(s)}(t)$ $=\left\langle\hat{\rho}_{\lambda \lambda^{\prime} \mathbf{K}_{\|}}^{(s s)}\right\rangle$, where $\langle\cdots\rangle$ has been meant by taking an expectation value; the diagonal element of $n_{\lambda \lambda^{\prime} \mathbf{K}_{\|}}^{(s)}(t)$ stands for the population density of the state with $\lambda$ and $\mathbf{K}_{\|}$in band $s$, while the off-diagonal element of $n_{\lambda \lambda^{\prime} \mathbf{K}_{\|}}^{(s)}(t)$ for $\lambda \neq \lambda^{\prime}$ represents the microscopic polarization of the intraband transition between the sites of $\lambda$ and $\lambda^{\prime}$ with $\mathbf{K}_{\|}$in band $s$.

Equation (9) for $p_{\lambda \lambda^{\prime} \mathbf{K}_{\|}}(t)$ given in the $\lambda$ representation (the Wannier representation) is of the standard form as the beginning expression of the semiconductor Bloch equations, similar to that in the $k$ representation widely used in the literature, ${ }^{12,42}$ where $k$ is the Bloch momentum of the SLs. Here, this is transformed into the equivalent equation for $p_{\mathbf{K}_{\|}}\left(z, z^{\prime}, t\right)$ in the $z$ representation for the purpose of employing the IDWSL Floquet basis set that will be described later [see Eq. (16) and the explanation following it], where $z$ and $z^{\prime}$ are the electronic coordinates in the direction of crystal growth. It is understood that the variable $O_{\lambda \lambda^{\prime}}$ in the $\lambda$ representation is connected with the alternative variable $O\left(z, z^{\prime}\right)$ through the relation

$$
O_{\lambda \lambda^{\prime}}=\int d z d z^{\prime}\langle\lambda \mid z\rangle O\left(z, z^{\prime}\right)\left\langle z^{\prime} \mid \lambda^{\prime}\right\rangle
$$

where $\langle z \mid \lambda\rangle$ is the Wannier function $w_{b}(z-l d)$ at the position $z-l d$, in miniband $b$ with $d$ as the lattice constant of the concerned SLs. By applying this to $p_{\lambda \lambda^{\prime} \mathbf{K}_{\|}}(t), O_{\lambda \lambda^{\prime}}$ and $O\left(z, z^{\prime}\right)$ are read as $p_{\lambda \lambda^{\prime} \mathbf{K}_{4}}(t)$ and $p_{\mathbf{K}_{\|}}\left(z, z^{\prime}, t\right)$, respectively. Similarly, for $n_{\lambda \lambda^{\prime} \mathbf{K}_{\|}}^{(s)}(t), Z_{\lambda \lambda^{\prime}}^{(s)}$, and $d_{\lambda \lambda^{\prime}}^{(c v)^{\prime}}$, these are also transformed into $n_{\mathbf{K}_{\|}}^{(s)}\left(z, z^{\prime}, t\right), Z^{(s)}\left(z, z^{\prime}\right)$, and $d^{(c v)}\left(z, z^{\prime}\right)$, respectively. Thus, Eq. (9) is recast into

$$
\begin{aligned}
i\left(\frac{d}{d t}\right. & \left.+\frac{1}{T_{2}}+i \epsilon_{\|}^{(v c)}\left(\mathbf{K}_{\|}\right)\right) p_{\mathbf{K}_{\|}}\left(z_{v}, z_{c}, t\right) \\
& =\int d z\left(\left[p_{\mathbf{K}_{\|}}\left(z_{v}, z, t\right) H^{(c)}\left(z, z_{c}, t\right)-H^{(v)}\left(z_{v}, z, t\right) p_{\mathbf{K}_{\|}}\left(z, z_{c}, t\right)\right]\right. \\
& -f_{p}(t)\left[n_{\mathbf{K}_{\|}^{(v)}}\left(z_{v}, z, t\right) d^{(v c)^{*}}\left(z, z_{c}\right)-d^{(v c)^{*}}\left(z_{v}, z\right) n_{\mathbf{K}_{\|}^{(c)}}^{\left.\left.\left(z, z_{c}, t\right)\right]\right),}\right.
\end{aligned}
$$

with $\epsilon_{\|}^{(v c)}\left(\mathbf{K}_{\|}\right)=\epsilon_{\|}^{(c)}\left(\mathbf{K}_{\|}\right)-\epsilon_{\|}^{(v)}\left(\mathbf{K}_{\|}\right)$. Here, $H^{(s)}\left(z, z^{\prime}, t\right)$ has been given by $\left\langle z\left|\hat{H}^{(s)}(t)\right| z^{\prime}\right\rangle$, where the operator $\hat{H}^{(s)}(t)$ is defined as

$$
\begin{aligned}
\hat{H}^{(s)}(t)= & \sum_{\lambda}\left[(-1)^{b+\sigma^{(s)}} \frac{\Delta_{b}^{(s)}}{4}\left(|\lambda\rangle\left\langle\lambda_{(+1)}|+| \lambda_{(+1)}\right\rangle\langle\lambda|\right)\right. \\
& \left.+\epsilon_{0 b}^{(s)}|\lambda\rangle\langle\lambda|\right]-F_{T}(t) \hat{Z}^{(s)} .
\end{aligned}
$$

Moreover, in this expression, $Z^{(s)}\left(z, z^{\prime}\right)$ is given by $\langle z| \hat{Z}^{(s)}$ $\times(t)\left|z^{\prime}\right\rangle$, where the operator $\hat{Z}^{(s)}(t)$ is defined as

$$
\hat{Z}^{(s)}=\frac{1}{2} \sum_{\lambda, \lambda^{\prime}}\left[|\lambda\rangle Z_{\lambda \lambda^{\prime}}^{(s)}\left\langle\lambda^{\prime}\right|+(\text { H.c. })\right] .
$$

The first term of Eq. (12) represents the Hamiltonian of the SL for the $s$ band by means of the NNTB model, and the second term of Eq. (12) stands for the dipole interaction with $F_{T}(t)$.

Bearing in mind that $f_{p}(t)$ is weak enough to approximate that $n_{\mathbf{K}_{\|}}^{(v)}\left(z, z^{\prime}, t\right)=\delta\left(z-z^{\prime}\right)$ and $n_{\mathbf{K}_{\|}^{(c)}}\left(z, z^{\prime}, t\right)=0$, Eq. (11) becomes

$$
\begin{aligned}
& e^{i \omega_{p} t} f_{p}(t) d^{(v c)^{*}}\left(z_{v}, z_{c}\right) \\
& \quad=\int d z\left[\bar{p}_{\mathbf{K}_{\|}}\left(z_{v}, z, t\right) H^{(c)}\left(z, z_{c}, t\right)-H^{(v)}\left(z_{v}, z, t\right) \bar{p}_{\mathbf{K}_{\|}}\left(z, z_{c}, t\right)\right] \\
& \quad-i\left(\frac{d}{d t}+\frac{1}{T_{2}}+i \epsilon_{\|}^{(v c)}\left(\mathbf{K}_{\|}\right)-i \omega_{p}\right) \bar{p}_{\mathbf{K}_{\|}}\left(z_{v}, z_{c}, t\right)
\end{aligned}
$$


where $p_{\mathbf{K}_{u}}\left(z_{v}, z_{c}, t\right)$ has been replaced by $e^{-i \omega_{p} t} \bar{p}_{\mathbf{K}_{u}}\left(z_{v}, z_{c}, t\right)$. This is the inhomogeneous equation corresponding to the homogeneous equation for the joint-miniband IDWSL Floquet wave functions $\psi_{\nu}\left(z_{v}, z_{c}, t\right)$, given by

$$
\begin{aligned}
\left(i \frac{d}{d t}+E_{\nu}\right) \psi_{\nu}\left(z_{v}, z_{c}, t\right)= & \int d z\left[\psi_{\nu}\left(z_{v}, z, t\right) H^{(c)}\left(z, z_{c}, t\right)-H^{(v)}\right. \\
& \left.\times\left(z_{v}, z, t\right) \psi_{\nu}\left(z, z_{c}, t\right)\right],
\end{aligned}
$$

with $E_{\nu}$ being the quasienergy and $\psi_{\nu}\left(z_{v}, z, t+T\right)$ $=\psi_{\nu}\left(z_{v}, z, t\right)$. Therefore, it is plausible to expand $\bar{p}_{\mathbf{K}_{\|}}\left(z_{v}, z_{c}, t\right)$ as

$$
\bar{p}_{\mathbf{K}_{\|}}\left(z_{v}, z_{c}, t\right)=\sum_{\nu} \psi_{\nu}\left(z_{v}, z_{c}, t\right) a_{\nu \mathbf{K}_{\|}}
$$

in terms of the IDWSL Floquet basis set $\left\{\psi_{\nu}\left(z_{v}, z_{c}, t\right)\right\}$. Using the orthonormality relation

$$
\frac{1}{T} \int_{0}^{T} d t \int d z_{v} d z_{c}\left[\psi_{\nu}\left(z_{v}, z_{c}, t\right)\right]^{*} \psi_{\nu^{\prime}}\left(z_{v}, z_{c}, t\right)=\delta_{\nu \nu^{\prime}}
$$

in Eq. (14) yields the explicit form of the expansion coefficient $a_{\nu_{\mathbf{K}_{\|}}}$as follows:

$$
\begin{aligned}
a_{\nu \mathbf{K}_{\|}}= & {\left[E_{\nu}+\epsilon_{\|}^{(v c)}\left(\mathbf{K}_{\|}\right)-\omega_{p}-i \gamma\right]^{-1} \frac{d_{0}^{(v c)^{*}}}{T} \int_{0}^{T} d t e^{i \omega_{p} t} f_{p}^{(+)} } \\
& \times(t) \int d z\left[\psi_{\nu}(z, z, t)\right]^{*},
\end{aligned}
$$

where $d^{(v c)}\left(z_{v}, z_{c}\right)$ has been given by $d_{0}^{(v c)} \delta\left(z_{v}-z_{c}\right)$, with $d_{0}^{(v c)}$ being the interband dipole transition matrix element of a bulk material, and $f_{p}(t)$ has been replaced by $f_{p}^{(+)}(t)$ $=\left(f_{p 0} / 2\right) e^{-i \omega_{p} t}$ since only photoabsorption is of concern here. It is remarked in Eq. (16) that the IDWSL Floquet basis set employed here is regarded as the more efficient one from the standpoint of numerical convergence in the actual simulation of Eq. (14) than the conventional basis sets in terms of the SL plane-wave function, ${ }^{12,42,43}$ the WSL wave function, ${ }^{44,45}$ and the DWSL Floquet wave function ${ }^{13}$ since the present basis set includes more physical information on Eq. (14) than the others. The above-mentioned transformation from the $\lambda$ representation to the $z$ representation is motivated by this statement as the first step to the IDWSL-Floquet representation of Eq. (18). ${ }^{46}$

The macroscopic polarization of the interband transition $P(t)$ is provided by

$$
\left.P(t)=\sum_{\lambda_{v}, \lambda_{c}, \mathbf{K}_{\|}, k} d_{\lambda_{v} \lambda_{c}}^{(v c)} p_{\lambda_{v} \lambda_{c} \mathbf{K}_{\|}}(t)+\text { (c.c. }\right),
$$

where $k$ is the Bloch momentum of the SLs that still remains conserved in the DWSL; therefore, this is implicitly included in $p_{\lambda_{v} \lambda_{c} \mathbf{K}_{\|}}(t)$ through $\left\{\psi_{\nu}\left(z_{v}, z_{c}, t\right)\right\}$. Further, (c.c.) means taking the complex conjugate of the first term. By employing Eqs. (16) and (18), Eq. (19) is recast into

$$
P(t)=\epsilon_{0} \chi(t) f_{p}^{(+)}(t)+(\text { c.c. }),
$$

where $\chi(t)$ corresponds to the linear optical susceptibility with respect to $f_{p}^{(+)}(t) \cdot \chi(t)$ is given by

$$
\chi(t)=\frac{1}{\epsilon_{0}}\left|d_{0}^{(v c)}\right|^{2} \sum_{\nu, \mathbf{K}_{\|, k}} \frac{\mathcal{O}_{\nu}(k, t)}{E_{\nu}+\epsilon_{\|}^{(v c)}\left(\mathbf{K}_{\|}\right)-\omega_{p}-i \gamma},
$$

where $\epsilon_{0}$ represents the dielectricity of vacuum and

$$
\mathcal{O}_{\nu}(k, t)=\sum_{\lambda_{v}, \lambda_{c}} o_{\lambda_{v} \lambda_{c}} \psi_{\lambda_{v} \lambda_{c} \nu}(t) \frac{1}{T} \int_{0}^{T} d t^{\prime} \int d z\left[\psi_{\nu}\left(z, z, t^{\prime}\right)\right]^{*} .
$$

In Eq. (22), it has been defined that

$$
\psi_{\lambda_{v} \lambda_{c} v}(t)=\int d z_{v} d z_{c}\left\langle\lambda_{v} \mid z_{v}\right\rangle \psi_{\nu}\left(z_{v}, z_{c}, t\right)\left\langle z_{c} \mid \lambda_{c}\right\rangle
$$

and

$$
o_{\lambda_{v} \lambda_{c}}=\int d z\left\langle\lambda_{v} \mid z\right\rangle\left\langle z \mid \lambda_{c}\right\rangle .
$$

Note that Eq. (21), along with Eq. (22), is the key expression to be developed in the following subsections and that its concise expression is mainly owing to the employment of the IDWSL Floquet basis set as stated before. It is remarked that the integration over $\mathbf{K}_{\|}$in Eq. (21) incurs the logarithmic divergence at $\left|\mathbf{K}_{\|}\right|=\infty$ due to the character inherent in the quasi-two-dimensional system without the exciton effect. To avoid such pathological behavior, hereafter, $\mathcal{O}_{\nu}(k, t)$ is implicitly read as $\mathcal{O}_{\nu}(k, t) e^{-\epsilon_{\|}^{(v c)}\left(\mathbf{K}_{\|}\right) \tau_{d} \text {, where the infinitesimal }}$ positive dumping constant $\tau_{d}$ has been introduced.

\section{B. Floquet states of the dynamic Wannier-Stark ladder}

In this subsection, Eq. (15) for $\psi_{\nu}\left(z_{v}, z_{c}, t\right)$ is solved for the further purpose of obtaining the explicit expression of $\chi(t)$ of Eq. (21) through Eq. (22). To do this, the Wannier function, $\langle z \mid \lambda\rangle=w_{b}(z-l d)$, is approximated by the corresponding wave function of a $\mathrm{QW}$ surrounded by infinite potential barriers. Henceforth, this model will be termed the infinite potential barrier QW (IPBQW) model. The criterion of the validity for this approximation is discussed in detail in Appendix A. For the sake of later convenience, $\hat{Z}^{(s)}$ of Eq. (13) is divided into the two terms as

$$
\hat{Z}^{(s)}=\hat{Z}_{0}^{(s)}+\hat{X}^{(s)},
$$

where $\hat{Z}_{0}^{(s)}$ and $\hat{X}^{(s)}$ are the intraminiband and interminiband interactions, respectively. By use of Eq. (A2) resulting from the IPBQW model, $\hat{Z}_{0}^{(s)}$ and $\hat{X}^{(s)}$ are expressed as

$$
\hat{Z}_{0}^{(s)}=\sum_{\lambda}|\lambda\rangle l d\langle\lambda|
$$

and

$$
\hat{X}^{(s)}=\frac{1}{2} \sum_{b, b^{\prime}(\neq b), l}\left[|b l\rangle X_{b b^{\prime}}^{(s)}\left\langle b^{\prime} l\right|+(\text { H.c. })\right]
$$

respectively, where $X_{b b^{\prime}}^{(s)}$ is given by Eq. (A3).

To solve Eq. (15) for $\psi_{v}\left(z_{v}, z_{c}, t\right)$, this wave function is expanded as 


$$
\psi_{\nu}\left(z_{v}, z_{c}, t\right)=\sum_{B, J} \Phi_{[B J]}^{*}\left(z_{v}, z_{c}, t\right) C_{[B J], \nu}
$$

where $\Phi_{[B J]}$ is the ZT-free joint-miniband DWSL Floquet wave function defined as

$$
\Phi_{[B J]}^{*}\left(z_{v}, z_{c}, t\right)=\phi_{b_{v} j_{v}}^{(v)}\left(z_{v}, t\right) \phi_{b_{c} j_{c}}^{(c)^{*}}\left(z_{c}, t\right),
$$

with $B=\left(b_{c}, b_{v}\right)$ and $J=\left(j_{c}, j_{v}\right)$. Hereafter, it is understood that the lump of the labels $B$ and $J$ is denoted as $[B, J]$ for specifying the ac-ZT-free joint-miniband DWSL Floquet state. In this equation, the single-miniband Floquet wave function $\phi_{b j}^{(s)}(z, t)$ is given by $\left\langle z \mid \phi_{b j}^{(s)}(t)\right\rangle$, with $\left|\phi_{b j}^{(s)}(t)\right\rangle$ represented by the Houston wave function ${ }^{16,47}$ as

$$
\left|\phi_{b j}^{(s)}(t)\right\rangle=e^{-i \hat{Z}_{0}^{(s)}[A(t) / c]} e^{i \varepsilon_{b j}^{(s)}(k) t-i \int^{t} d t^{\prime} \epsilon_{b}^{(s)}\left(k-A\left(t^{\prime}\right) / c\right)}|b k\rangle,
$$

where $|b k\rangle$ represents the SL state with the energy dispersion $\epsilon_{b}^{(s)}(k)$ of the miniband $b$ at the joint Bloch momentum $k .|b k\rangle$ and $\epsilon_{b}^{(s)}(k)$ are given by

$$
|b k\rangle=\frac{1}{\sqrt{N_{q w}}} \sum_{l} e^{i k l d}|b l\rangle,
$$

with $N_{q w}$ being the number of QWs included in the SLs, and

$$
\epsilon_{b}^{(s)}(k)=\left[\epsilon_{0 b}^{(s)}+(-1)^{b+\sigma^{(s)}} \frac{\Delta_{b}^{(s)}}{2} \cos (k d)\right],
$$

respectively. In Eq. (30), $A(t)$ is the vector potential associated with $F_{T}(t): F_{T}(t)=-\dot{A}(t) / c$, with $c$ as the speed of light. Note that $\left|\phi_{b j}^{(s)}(t)\right\rangle$ satisfies

$$
\left[i \frac{d}{d t}+\varepsilon_{b j}^{(s)}(k)-\hat{H}_{0}^{(s)}(t)\right]\left|\phi_{b j}^{(s)}(t)\right\rangle=0,
$$

where

$$
\begin{aligned}
\hat{H}_{0}^{(s)}(t)= & \sum_{\lambda}\left[(-1)^{b+\sigma^{(s)}} \frac{\Delta_{b}^{(s)}}{4}\left(|\lambda\rangle\left\langle\lambda_{(+1)}|+| \lambda_{(+1)}\right\rangle\langle\lambda|\right)\right. \\
& \left.+\epsilon_{0 b}^{(s)}|\lambda\rangle\langle\lambda|\right]-F_{T}(t) \hat{Z}_{0}^{(s)}
\end{aligned}
$$

Further, $\varepsilon_{b j}^{(s)}(k)$ is the quasienergy of the $j$ th photon sideband of miniband $b$, that is, $\varepsilon_{b j}^{(s)}(k)=\varepsilon_{b 0}^{(s)}(k)+j \omega$, with $\varepsilon_{b 0}^{(s)}(k)$ being the quasienergy of the parent band. Using Eqs. (30) and (31), Eq. (29) is recast into

$$
\begin{aligned}
& \Phi_{[B J]}^{*}\left(z_{v}, z_{c}, t\right) \\
& =e^{-i\left(z_{v}-z_{c}\right)[A(t) / c]} e^{-i \varepsilon_{[B J]}(k) t+i \int^{t} d t^{\prime} \epsilon_{B}\left(k-A\left(t^{\prime}\right) / c\right)} \varphi_{B k}^{*}\left(z_{v}, z_{c}\right),
\end{aligned}
$$

where $\varepsilon_{[B J]}(k)=\varepsilon_{b_{c} 0}^{(c)}(k)-\varepsilon_{b_{v} 0}^{(v)}(k)+J \omega$, with $J$ redefined as $J$ $=j_{c}-j_{v}$, and $\epsilon_{B}(k)=\epsilon_{b_{c}}^{(c)}(k)-\epsilon_{b_{v}}^{(v)}(k) . \varphi_{B k}\left(z_{v}, z_{c}\right)$ is the jointminiband SL wave function given by

$$
\varphi_{B k}\left(z_{v}, z_{c}\right)=\frac{1}{\sqrt{N_{q w}}} \sum_{l} e^{i k l d} W_{B l}\left(z_{v}, z_{c}\right),
$$

where

$$
W_{B l}\left(z_{v}, z_{c}\right)=\frac{1}{\sqrt{N_{q w}}} \sum_{l^{\prime}}\left\langle b_{v} l^{\prime} \mid z_{v}\right\rangle\left\langle z_{c} \mid b_{c} l+l^{\prime}\right\rangle .
$$

Inserting Eq. (28) into Eq. (15) in view of Eqs. (33)-(37) yields the following eigenvalue equation for a set of the expansion coefficients $\left\{C_{[B J], \nu}\right\}$ :

$$
\left[E_{\nu}-\varepsilon_{[B J]}(k)\right] C_{[B J], \nu}=-\sum_{B^{\prime} J^{\prime}} \mathcal{V}_{[B J],\left[B^{\prime} J^{\prime}\right]} C_{\left[B^{\prime} J^{\prime}\right], \nu}
$$

Here,

$$
\mathcal{V}_{[B J],\left[B^{\prime} J^{\prime}\right]}=\left(X_{b_{c} b_{c}^{\prime}}^{(c)} \delta_{b_{v} b_{v}^{\prime}}-X_{b_{v} b_{v}^{\prime}}^{(v)} \delta_{b_{c} b_{c}^{\prime}}\right) \mathcal{F}_{[B J],\left[B^{\prime} J^{\prime}\right]},
$$

where

$$
\begin{aligned}
& \mathcal{F}_{[B J],\left[B^{\prime} J^{\prime}\right]}=\frac{1}{T} \int_{0}^{T} d t F_{T}(t) \\
& \times d e^{i\left[\varepsilon_{[B J]}(k)-\varepsilon_{\left[B^{\prime} J^{\prime}\right]}(k)\right] t-i \int^{t} d t^{\prime}\left[\epsilon_{B}\left(k-A\left(t^{\prime}\right) / c\right)-\epsilon_{B^{\prime}}\left(k-A\left(t^{\prime}\right) / c\right)\right]} .
\end{aligned}
$$

By adopting the high-frequency approximation, ${ }^{16}$ Eq. (40) becomes independent of $B$ and $B^{\prime}$, leading to

$$
\mathcal{F}_{[B J],\left[B^{\prime} J^{\prime}\right]} \equiv \frac{\omega}{d} V_{\Delta J}=\frac{1}{T} \int_{0}^{T} d t F_{T}(t) e^{i \Delta J \omega t},
$$

where $\Delta J=J-J^{\prime}$. This approximation is equivalent to the replacement of the exponent of Eq. (35), $\varepsilon_{[B J]}(k)-\int^{t} d t^{\prime} \epsilon_{B}(k$ $\left.-\frac{A\left(t^{\prime}\right)}{c}\right)$, by $J \omega t$. The validity of this approximation is mentioned in Appendix A. Using the explicit form of $F_{T}(t), V_{\Delta J}$ becomes

$$
\begin{aligned}
V_{\Delta J}= & \frac{\Omega_{\mathrm{Bloch}}^{\prime}}{\omega} \delta_{\Delta J 0}-\frac{Z}{2 \pi}\left\{e^{i \delta} \sin [(x-\Delta J) \pi \mu]\right. \\
& \left.+e^{-i \delta} \sin [(x+\Delta J) \pi \mu]\right\}+\frac{1}{2} Z x \mu\left\{e^{i \delta} j_{0}[(x-\Delta J) \pi \mu]\right. \\
& \left.+e^{-i \delta} j_{0}[(x+\Delta J) \pi \mu]\right\}
\end{aligned}
$$

where $Z=F_{a c} d / \Omega$ and $j_{0}(\xi)$ is the zeroth-order spherical Bessel function of the first kind. Further, $\Omega_{\text {Bloch }}^{\prime}$ is the effective Bloch frequency defined by

$$
\Omega_{\mathrm{Bloch}}^{\prime}=F_{d c}^{\prime} d=\Omega_{\mathrm{Bloch}}+\frac{Z \omega}{\pi} \sin (\pi x \mu) \cos \delta,
$$

where $\Omega_{\text {Bloch }}$ is the Bloch frequency given by $\Omega_{\text {Bloch }}=F_{d c} d$. In the first equality of Eq. (43), $F_{d c}^{\prime}$ is the effective dc field, defined by

$$
F_{d c}^{\prime}=F_{d c}+\left(F_{a c} / \pi x\right) \sin (\pi x \mu) \cos \delta,
$$

where the second term arises from the stationary contribution of $F(t)$. In Eq. (42), the first term contributes to the dc-ZT only when $\Delta J=0$, while the second and third terms contribute to the ac-ZT when $\Delta J \neq 0$. Equation (38) is solved numerically using Eqs. (39)-(43). 


\section{Effective absorption coefficient}

Once a set of the Floquet wave functions $\left\{\psi_{\nu}\right\}$ of Eq. (28) is provided, the macroscopic polarization $P(t)$ of Eq. (20) is calculated by using Eq. (21). In the present subsection, one seeks for the explicit expression of the effective absorption coefficient $\alpha_{a b s}\left(\omega_{p} ; \omega\right)$ with respect to the probe light $f_{p}(t)$. This is defined as

$$
\alpha_{a b s}\left(\omega_{p} ; \omega\right)=-\frac{1}{I(z)} \frac{d I(z)}{d z},
$$

where $I(z)$ and $d I(z) / d z$ represent the intensity of the probe light at depth $z$ of a sample from the surface and its derivative with respect to $z$, respectively. ${ }^{48}$ It is noted again that the PPT field $F(t)$ does not affect the interband transition due to $\omega_{p} \gg \omega$, as mentioned previously. Further, it is assumed that the degree of magnitude of $P(t)$ is negligibly smaller than $\epsilon_{0} f_{p 0}$. Therefore, $I(z)$ and $d I(z) / d z$ are approximated as $I(z)$ $\approx I(0)=\frac{1}{2} \epsilon_{0} c f_{p 0}^{2}$, and

$$
\left.\frac{d I(z)}{d z} \approx \frac{d I(z)}{d z}\right|_{z=0}=-\frac{1}{T_{p}} \int_{0}^{T_{p}} d t f_{p}(t) \frac{\partial P(t)}{\partial t},
$$

where $T_{p}=2 \pi / \omega_{p}$.

On the other hand, using Eqs. (28) and (35), Eq. (22) is reduced to

$$
\mathcal{O}_{\nu}(k, t)=\sum_{J} e^{i J \omega t} \widetilde{\mathcal{O}}_{J \nu}(k)
$$

where

$$
\widetilde{\mathcal{O}}_{J \nu}(k)=\mathcal{C}_{-J \nu}(k) \mathcal{C}_{0 \nu}^{*}(k) .
$$

Further, $\mathcal{C}_{J \nu}(k)$ has been defined as

$$
\mathcal{C}_{J \nu}(k)=\sum_{B} C_{[B J], \nu}(k) o_{B}^{(v c)}
$$

where $o_{B}^{(v c)}=o_{\left(b_{v} 0\right),\left(b_{c} 0\right)} \approx \delta_{b_{v} b_{c}}$, using the definition of Eq. (24) in the first equality and the IPBQW approximation in the second equality. Then, Eq. (21) becomes

$$
\chi(t)=\sum_{J} e^{i J \omega t} \chi_{J}\left(\omega_{p} ; \omega\right)
$$

where

$$
\chi_{J}\left(\omega_{p} ; \omega\right)=\frac{1}{\epsilon_{0}}\left|d_{0}^{(v c)}\right|^{2} \sum_{\nu, \mathbf{K}_{\|, k}} \frac{\widetilde{\mathcal{O}}_{J \nu}(k)}{E_{\nu}(k)+\epsilon_{\|}^{(v c)}\left(\mathbf{K}_{\|}\right)-\omega_{p}-i \gamma} .
$$

Here, the $k$ dependence of $C_{[B J], \nu}$ and $E_{\nu}$ have been explicitly specified. Obviously, $\widetilde{\mathcal{O}}_{J \nu}(k)$ represents the correction factor attributed to the ZTs to the strength of the interband bulk transition $\left|d_{0}^{(v c)}\right|^{2}$. Unless there are ZTs, $\nu$ is specified, for example, as $\left[B_{0}, J_{0}\right]$ and hence $\widetilde{\mathcal{O}}_{J \nu}(k)$ just becomes $\delta_{J J_{0}} \delta_{J 0}\left|o_{B_{0}}^{(v c)}\right|^{2}$. Therefore, Eq. (51) is reduced to the conventional form ${ }^{13}$

$$
\chi_{J}\left(\omega_{p} ; \omega\right)=\delta_{J 0} \frac{1}{\epsilon_{0}}\left|d_{0}^{(v c)}\right|^{2} \sum_{B_{0}, \mathbf{K}_{\|}, k} \frac{\left|o_{B_{0}}^{(v c)}\right|^{2}}{\varepsilon_{\left[B_{0} 0\right]}(k)+\epsilon_{\|}^{(v c)}\left(\mathbf{K}_{\|}\right)-\omega_{p}-i \gamma} .
$$

Owing to the employment of the IDWSL-Floquet basis set [see Eq. (16)], $\chi_{J}\left(\omega_{p} ; \omega\right)$ becomes of the form of Eq. (51) comparable with Eq. (52). Such an analytical and close expression is very helpful to understand the underlying physics relevant to the ZT since this is incorporated just in $\widetilde{\mathcal{O}}_{J \nu}(k)$ and $E_{\nu}(k)$ in Eq. (51) in place of $\left|o_{B_{0}}^{(v c)}\right|^{2}$ and $\varepsilon_{\left[B_{0} 0\right]}(k)$ in Eq. (52), respectively. In other words, ZT affects the absorption intensity through the factor of $\widetilde{\mathcal{O}}_{J \nu}(k)$ along with the absorption position $E_{\nu}(k)$. Without the use of the present basis set, the resulting equation for $\chi_{J}\left(\omega_{p} ; \omega\right)$ that corresponds to Eq. (51) cannot avoid being evaluated in a fully numerical manner.

From Eqs. (20) and (50), Eq. (45) becomes of the form

$$
\begin{aligned}
\alpha_{a b s}\left(\omega_{p} ; \omega\right)= & \frac{\omega_{p}}{c} \sum_{J} \operatorname{Im}\left[\chi_{J}\left(\omega_{p} ; \omega\right)(1-J \Delta)(D(-2, J)\right. \\
& +D(0, J))] \\
& \approx \frac{\omega_{p}}{c} \sum_{J} \operatorname{Im} \chi_{J}\left(\omega_{p} ; \omega\right)
\end{aligned}
$$

where

$$
D(n, J)=e^{i \pi(n+J \Delta)} j_{0}[\pi(n+J \Delta)],
$$

with $\Delta=\omega / \omega_{p}$, and the approximation $D(n, J) \approx \delta_{n 0}$ has been made in Eq. (54) since $\Delta \ll 1$ holds correctly, as demonstrated in Appendix A. Hereafter, the approximation of $\Delta$ $\approx 0$ is used throughout, unless otherwise stated. Equations (51) and (54) are the central results obtained in Sec. II.

\section{RESULTS AND DISCUSSION}

The calculated results of the quasienergy $E_{\nu}(k)$, given by Eq. (38), and the effective absorption coefficient $\alpha_{a b s}\left(\omega_{p} ; \omega\right)$, given by Eq. (54), are presented, followed by a discussion. The calculations have been done within the four jointminiband model, in which just the joint minibands labeled as $B=(1,1),(1,2),(2,1)$, and $(2,2)$ are included, where the definition of $B$ is given right below Eq. (29). The sample of the SLs for the calculations is $\mathrm{GaAs} / \mathrm{Ga}_{0.75} \mathrm{Al}_{0.25} \mathrm{As}$ of $35 / 11$ ML (monolayers) $[1 \mathrm{ML}=2.83 \AA]$ for the well and barrier thicknesses. Here, the barrier heights of the confining potentials of electrons in the $c$ and $v$ bands are 212.0 and $99.8 \mathrm{meV}$, respectively. The material parameters of $m_{\|}^{(c)}$, $m_{\|}^{(v)}$, and $E_{g}$ are given in Ref. 49. Moreover, the jointminiband center $\epsilon_{0 B}^{(v c)}$ and width $\left|\Delta_{B}^{(v c)}\right|$ are given in Table I, where these are defined as $\epsilon_{0 B}^{(v c)}=\epsilon_{0 b_{c}}^{(c)}-\epsilon_{0 b_{v}}^{(v)}$ and $\Delta_{B}^{(v c)}$ $=(-1)^{b_{c}} \Delta_{b_{c}}^{(c)}+(-1)^{b_{v}} \Delta_{b_{v}}^{(v)}$. The homogeneous broadening of $\gamma$ $=1 \mathrm{meV}$ is used for the calculations of $\alpha_{a b s}\left(\omega_{p} ; \omega\right)$ and $\alpha_{a b s}^{0}\left(\omega_{p} ; \omega\right)$. Further, the case where the effective dc field $F_{d c}^{\prime}$ vanishes is exclusively considered here, unless otherwise 
TABLE I. The adopted parameters of the joint-miniband center $\epsilon_{0 B}^{(v c)}(\mathrm{eV})$ reckoned from the bottom of the $c$ band and the jointminiband width $\left|\Delta_{B}^{(v c)}\right|(\mathrm{meV})$ for the SLs of $35 \mathrm{ML} \mathrm{GaAs} / 11 \mathrm{ML}$ $\mathrm{Ga}_{0.75} \mathrm{Al}_{0.25} \mathrm{As}$.

\begin{tabular}{lcc}
\hline \hline$B=\left(b_{c}, b_{v}\right)$ & $\epsilon_{0 B}^{(v c)}$ & $\left|\Delta_{B}^{(v c)}\right|$ \\
\hline$(1,1)$ & 1.557 & 8.82 \\
$(2,1)$ & 1.650 & 37.55 \\
$(1,2)$ & 1.579 & 10.63 \\
$(2,2)$ & 1.672 & 39.36 \\
\hline \hline
\end{tabular}

stated. This choice follows the criterion of the validity for the IPBQW approximation discussed in Appendix A; that is, this approximation is not suitable for the case that the resonant dc-ZT is dominant. Thus, the system concerned becomes akin to the SLs subject to the dynamic Franz-Keldysh effect, ${ }^{22}$ aside from contributions of the ac-ZT between subbands.

Hereafter, the IDWSL driven by the two types of the total electric field $F_{T}(t)$ with $F_{d c}^{\prime}=0$ is examined; one is the PPT with $\mu=1$ and $\delta=\pi / 2$, and the other is the PPT with $\mu$ $=0.5$ and $\delta=\pi$. The former and latter PPTs are termed PPT- 1 and PPT-2, respectively. In Fig. 2, the wave forms of the PPT-1 and PPT-2 are shown for $x=0.1,0.5$, and 0.9 in panels (a) and (b), respectively. It is remarked that the wave forms of the PPT- 1 and PPT- 2 with $x=0.1$ are almost saw-toothed and square, respectively. It is worth mentioning that the present choice of the PPT-1 and PPT-2 makes it possible to keep track of the modulation patterns of the spectra from one region where the coherent removal of the ac-ZT is dominant to the other region where the spectral anomaly manifests itself due to the ac-ZT by changing $x$ in a systematic manner.

(a) PPT-1

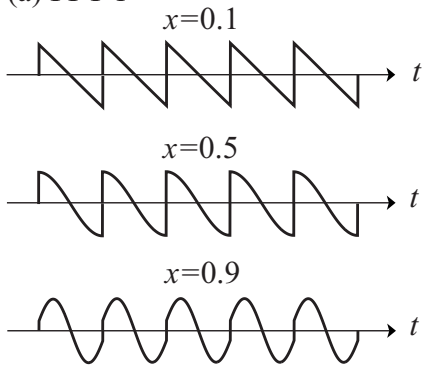

(b) PPT-2

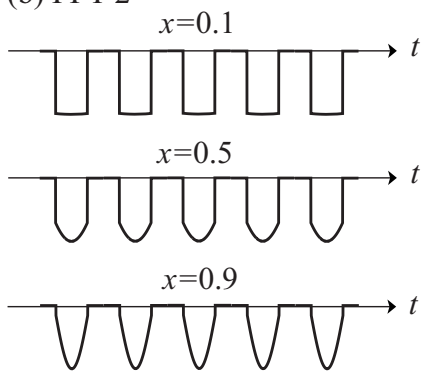

FIG. 2. The wave forms of (a) the PPT-1 $(\mu=1, \delta=\pi / 2)$ and (b) the PPT-2 $(\mu=0.5, \delta=\pi)$ for $x=0.1,0.5$, and 0.9 .

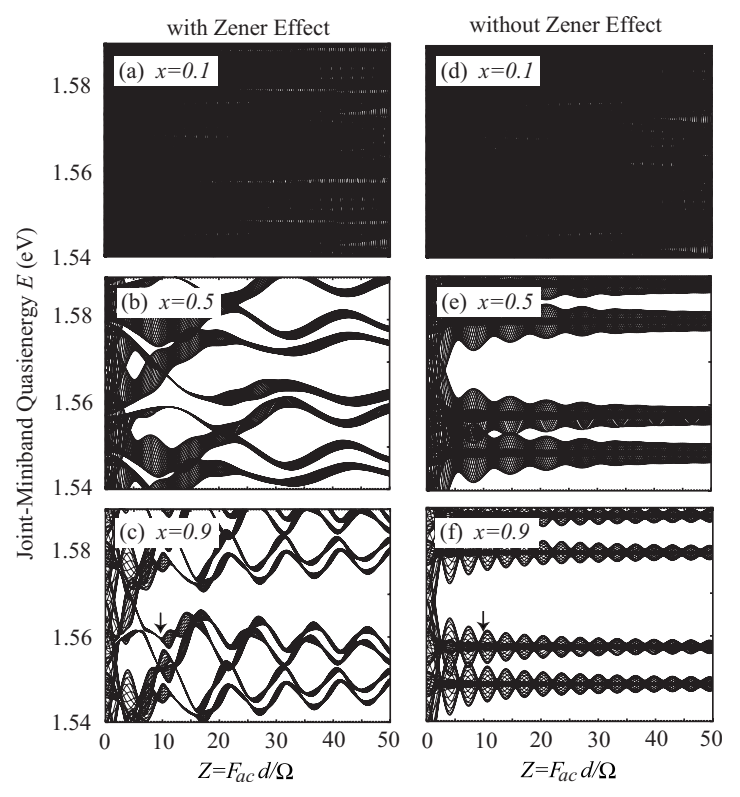

FIG. 3. The joint-miniband quasienergies $E_{\nu}(k)$ as a function of $Z=F_{d c} d / \Omega$ for the PPT- 1 with $x=0.1,0.5$, and 0.9 and $N_{\omega}=3$. The quasienergies depicted in panels (a)-(c) are obtained with the acZT, while those in panels (d)-(f) are obtained without the ac-ZT. The arrowed portions in panels (c) and (f) at $Z=10$ are used to aid the discussion made in Sec. III C. For more details, consult the text.

Of course, other types of PPTs can be adopted straightforward in the present calculations, even if $F_{d c}^{\prime} \neq 0$ as long as the dc-ZT is less dominant; the resulting spectra also show the alteration from the ac-ZT-free reference spectra similar to that observed in the cases of the PPT- 1 and PPT- 2 in proportion to the strength of the ac-ZT, though not shown here. However, there are a lot of possible combinations of the PPT parameters to determine a wave form, and hence a guide of choosing specific wave forms is requisite in practice; the systematic inspection of the effect of the ac-ZT guides us toward the present choice of the PPTs.

This section is organized as follows. In Sec. III A, $E_{\nu}(k)$ 's driven by the PPT- 1 and PPT-2 are presented, followed by a comparison with the case without the ac-ZT. In Sec. III B, the spectra $\alpha_{a b s}\left(\omega_{p} ; \omega\right)$ associated with $E_{\nu}(k)$ 's shown in the preceding subsection are presented, where the anomalous modulation pattern of the spectra is pointed out. Moreover, the exciton effect on $\alpha_{a b s}\left(\omega_{p} ; \omega\right)$ is also mentioned. In Sec. III C, the differential effective absorption coefficient, $\alpha_{a b s}^{0}\left(\omega_{p} ; \omega\right) \propto \partial \alpha_{a b s}\left(\omega_{p} ; \omega\right) / \partial \omega_{p}$, is introduced in order to develop the discussion on the anomaly of $\alpha_{a b s}\left(\omega_{p} ; \omega\right)$ and the exciton effect mentioned in the preceding subsection. In Sec. III D, the origin of this anomaly is explored. In Sec. III E, the miscellaneous results of the concerned spectra are presented.

\section{A. Quasienergies}

Figures 3 and 4 show $E_{\nu}(k)$ as a function of $Z=F_{a c} d / \Omega$ for the PPT-1 and PPT-2, respectively, for $x=0.1,0.5$ and 0.9 , where the ZT-free quasienergies, that is, $\varepsilon_{[B J]}(k)=\varepsilon_{b_{c} 0}^{(c)}(k)$ $-\varepsilon_{b_{v} 0}^{(v)}(k)+J \omega$, are also depicted for comparison. Here, using 

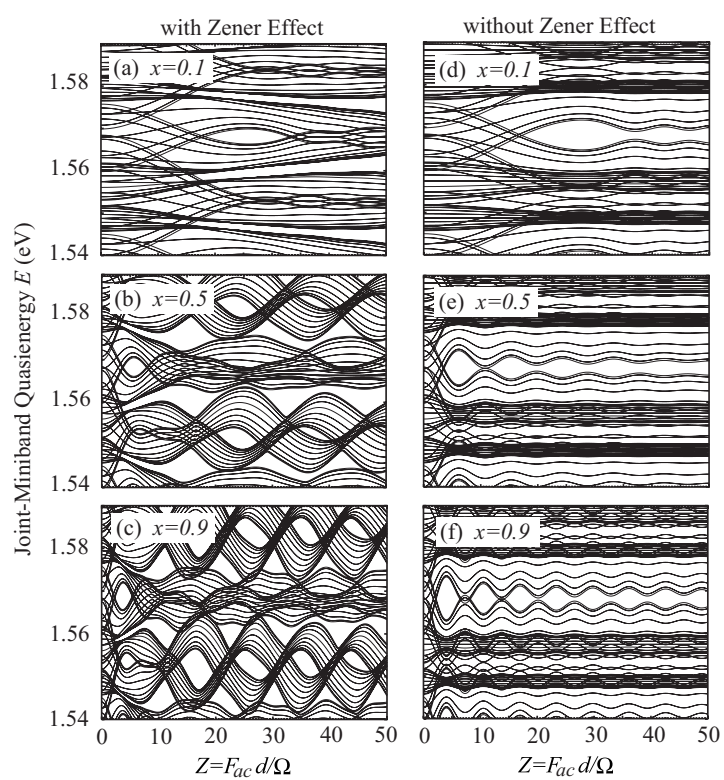

FIG. 4. The same as Fig. 3 but for the PPT-2.

Eq. (32), the explicit form of $\varepsilon_{b 0}^{(s)}(k)$ is given by the temporal average of the instantaneous energy $\epsilon_{b}^{(s)}[k-A(t) / c]$ in the following:

$$
\begin{aligned}
\varepsilon_{b 0}^{(s)}(k)= & \frac{1}{T} \int_{0}^{T} d t \epsilon_{b}^{(s)}\left(k-\frac{A(t)}{c}\right) \\
= & {\left[\epsilon_{0 b}^{(s)}+(-1)^{b+\sigma^{(s)}} \frac{\Delta_{b}^{(s)}}{2}\left\{\cos (k d)\left[\delta_{N 0}-\mu j_{0}(N \mu \pi)\right]\right.\right.} \\
& \left.\left.+\mu \sum_{l=-\infty}^{\infty} J_{l}(Z) \cos (k d+l \delta) j_{0}([N+l x] \mu \pi)\right\}\right],
\end{aligned}
$$

where $N=0$ in the present case of $F_{d c}^{\prime}=0$, though this integer $N$ is defined in general as the resonant condition $N$ $=\Omega_{\text {Bloch }}^{\prime} / \omega$. In addition, the value of $N_{\omega}$ is defined as the ratio of the energy difference of the miniband centers between $B$ $=\left(2, b_{v}\right)$ and $\left(1, b_{v}\right)$ to $\omega$, namely, $N_{\omega}=\left[\epsilon_{0\left(2, b_{v}\right)}^{(v c)}-\epsilon_{0\left(1, b_{v}\right)}^{(v c)}\right] / \omega$. In Figs. 3 and 4, $N_{\omega}$ has been set equal to 3 , and thusj the quasienergy forms a repeated photon sideband in every $\omega=0.031 \mathrm{eV}$. In this situation, the energy center of $\varepsilon_{\left[\left(1, b_{v}\right) J\right]}(k)$ coincides with that of $\varepsilon_{\left[\left(2, b_{v}\right)\left(J-N_{\omega}\right)\right]}(k)$, and this resonant condition gives rise to the dominant ac-ZT between the ZT-free states, $\Phi_{\left[\left(1, b_{v}\right) J\right]}$ and $\Phi_{\left[\left(2, b_{v}\right)\left(J-N_{\omega}\right)\right]}$, leading to a strong anticrossing.

The quasienergies of Figs. 3(a) and 4(a) are almost identical to those of Figs. 3(d) and 4(d), respectively, and it is speculated that the ac-ZT has little effect on $E_{\nu}(k)$. This is due to the coherent removal of the ac-ZT by means of the PPT-1 and PPT-2 with $x \ll 1$, the wave form of which are saw-toothed and square, respectively, as seen in Fig. 2; in fact, $V_{\Delta J}$ of Eq. (42) almost vanishes in these cases. ${ }^{35}$ Every lobe of $E_{\nu}(k)$ in Figs. 3(a) and 3(d) is approximately expressed by use of Eq. (57) as

$$
E_{[B, J]}(k)=\epsilon_{0 B}^{(v c)}+\frac{\Delta_{B}^{(v c)}}{2} \cos (k d+Z)+J \omega,
$$

where index $\nu$ has been specified as $[B, J]$. It is seen that the width of $E_{[B, J]}(k)$, namely, $\left|\Delta_{B}^{(v c)}\right|$, is independent of $Z$ and is exactly the similar to that of the original SLs. Therefore, the DL, which is characteristic of the DWSL driven by the monochromatic and bichromatic laser fields, is always absent from this case, and the complete DDL manifests itself over the entire region of Z. As seen in Figs. 3(a) and 3(d), the resulting quasienergy fills the whole space of the figures since every lobe of the quasienergy with the maximum width featured by the complete DDL is partially overlapped with adjacent lobes because of $N_{\omega}=3$. Next, as regards the lobe of $E_{\nu}(k)$ in Figs. 4(a) and 4(d), this is approximately expressed by use of Eq. (57) as

$$
E_{[B, J]}(k)=\epsilon_{0 B}^{(v c)}+\frac{\Delta_{B}^{(v c)}}{4}\left[1+j_{0}(\pi Z x / 2)\right] \cos (k d)+J \omega .
$$

The width is given by $\left|\Delta_{B}^{(v c)}\right|\left[1+j_{0}(\pi Z x / 2)\right] / 2$, and it becomes $\left|\Delta_{B}^{(v c)}\right| / 2$ with increasing $Z$. Hence, similar to Eq. (58), the quasienergy is characterized by the DDL, and the DL does not appear at all. In general, for the square-wave PPT $(x \ll 1)$ with $N=0$ and an arbitrary value of $\mu=\tau / T$, if $F_{a c} \gg 1$, the quasienergy becomes similar to that of the original SLs besides the width being reduced by $1-\mu$. This result ascribes to the integration of Eq. (56) in the interval, $[\tau / 2, T-\tau / 2]$, in which the laser is off, as shown in Eqs. (1) and (2). Therefore, it would be concluded that no effect on the SLs is caused by irradiation of the periodic $\delta$-pulse train (with $F_{a c} \gg 1$ and $\mu \ll 1$ ) as a special case, though this pulse plays decisive roles in diverse phenomena, for instance, in the chaos manifesting itself in a periodically kicked atom. ${ }^{50}$

Meanwhile, as $x$ increases from that of Figs. 3(a) and 4(a), $E_{\nu}(k)$ 's in Figs. 3(b), 3(c), 4(b), and 4(c) show more complicated structures than those in Figs. 3(e), 3(f), 4(e), and 4(f), respectively, since the ac-ZT can no longer be removed by the PPT-1 and PPT-2 concerned. Indeed, the examination of the alteration patterns of the quasienergies is important as the initial characterization of the IDWSL Floquet states. However, it seems difficult to gain deeper insight into the underlying physics in this region of $x$ simply by examining the quasienergies. Therefore, the further analyses of the effect of the ac-ZT are deferred to the subsequent subsections regarding the effective absorption spectra, where the modulation of the quasienergy shown here is consulted again.

\section{B. Effective absorption coefficient: $\alpha_{a b s}\left(\omega_{p} ; \omega\right)$}

First, the spectral structure of the effective absorption coefficient $\alpha_{a b s}\left(\omega_{p} ; \omega\right)$ is surveyed. If the limit of $\gamma \rightarrow 0$ is taken in Eq. (51), the imaginary part of it becomes 


$$
\begin{gathered}
\operatorname{Im} \chi_{J}\left(\omega_{p} ; \omega\right)=\frac{1}{\epsilon_{0}}\left|d_{0}^{(v c)}\right|^{2} \sum_{\nu, \mathbf{K}_{\|,},}\left(\pi \operatorname { R e } [ \widetilde { \mathcal { O } } _ { J \nu } ( k ) ] \delta \left(E_{\nu}(k)+\epsilon_{\|}^{(v c)}\right.\right. \\
\left.\left.\times\left(\mathbf{K}_{\|}\right)-\omega_{p}\right)+P \frac{\operatorname{Im}\left[\widetilde{\mathcal{O}}_{J \nu}(k)\right]}{E_{\nu}(k)+\epsilon_{\|}^{(v c)}\left(\mathbf{K}_{\|}\right)-\omega_{p}}\right)(60) \\
\approx \frac{\pi}{\epsilon_{0}}\left|d_{0}^{(v c)}\right|^{2} \mathcal{D}_{\|} \sum_{\nu, k} \operatorname{Re}\left[\widetilde{\mathcal{O}}_{J \nu}(k)\right] \Theta\left(\omega_{p}-E_{\nu}(k)\right),
\end{gathered}
$$

where the symbol $P$ means to take the Cauchy principal value, and the second equality is provided under the approximation that the on-the-energy-shell contribution of the first term in the parentheses of Eq. (60) is usually much dominant to the off-the-energy-shell contribution of the second one in the vicinity of $\omega_{p}=E_{\nu}(k)$. Here, $\mathcal{D}_{\|}$is the constant representing the two-dimensional density of state for the free electronic motion on the plane parallel to the layer plane; this motion is associated with $\mathbf{K}_{\|}$. On the other hand, the threedimensional density of state of the present system is given by

$$
\mathcal{D}\left(\omega_{p}\right)=\mathcal{D}_{\|} \sum_{\nu, k} \Theta\left[\omega_{p}-E_{\nu}(k)\right] .
$$

This shows the simple ascending steplike structure. By comparing Eq. (61) with this, it is obviously seen that aside from the weight factor $\operatorname{Re}\left[\widetilde{\mathcal{O}}_{J \nu}(k)\right]$ for the correction due to the ac-ZT, $\alpha_{a b s}\left(\omega_{p} ; \omega\right)$ reflects this density of state for the IDWSL Floquet state. Therefore, it is expected that such an ascending steplike structure is more or less modified by introducing the ac-ZT through $\operatorname{Re}\left[\widetilde{\mathcal{O}}_{J \nu}(k)\right]$. Without the ac-ZT, the profile of $\alpha_{a b s}\left(\omega_{p} ; \omega\right)$ would remain ascending steplike because of $\left|o_{B_{0}}^{(v c)}\right|^{2} \geqslant 0$, where $\operatorname{Re}\left[\widetilde{\mathcal{O}}_{J \nu}(k)\right]$ is replaced by $\left|o_{B_{0}}^{(v c)}\right|^{2} \delta_{J_{0}} \delta_{J 0}$ in Eq. (61) with $\nu \equiv\left[B_{0}, j_{0}\right]$. It is remarked that regardless of the ac-ZT, each profile of the IDWSL-Floquet state in $\alpha_{a b s}\left(\omega_{p} ; \omega\right)$ is superimposed on the ascending steplike structure resulting from the accumulation of other states with smaller quasienergies; in other words, the spectral profiles of these Floquet states are not separated from each other.

Next, bearing in mind the general view of $\alpha_{a b s}\left(\omega_{p} ; \omega\right)$, the spectral modulation with respect to $x$ is examined. In Figs. 5 and $6, \alpha_{a b s}\left(\omega_{p} ; \omega\right)$, defined in Eq. (54) using Eq. (51), of the IDWSL driven by the PPT-1 and PPT-2, respectively, are shown by the red curves for $x=0.1-0.9$ with $Z=10$ and $N_{\omega}$ $=3$. For the purpose of comparison, the corresponding effective absorption coefficients without the ac-ZT are also depicted by the blue curves, where Eq. (52) is used in Eq. (54). Here, $\alpha_{a b s}\left(\omega_{p} ; \omega\right)$ is read as the relative value, $\alpha_{a b s}\left(\omega_{p} ; \omega\right)$ $-\alpha_{a b s}\left(E_{g} ; \omega\right)$, due to the logarithmic divergence mentioned right below Eq. (24). As expected from the discussion in Figs. 3 and 4, the spectral profiles for $x=0.1$ indicate the sequence of the ascending steplike structure due to the coherent removal of the ac-ZT. The first and second steps seen in Fig. 5 for $x=0.1$ around $\omega_{p}=1.56$ and $1.68 \mathrm{eV}$ correspond to the Floquet states of the parent bands with the lobes $E_{[(1,1), 0]}(k)$ and $E_{[(2,2), 0]}(k)$, respectively, given by Eq. (58). On the other hand, there are no contributions from the pho-

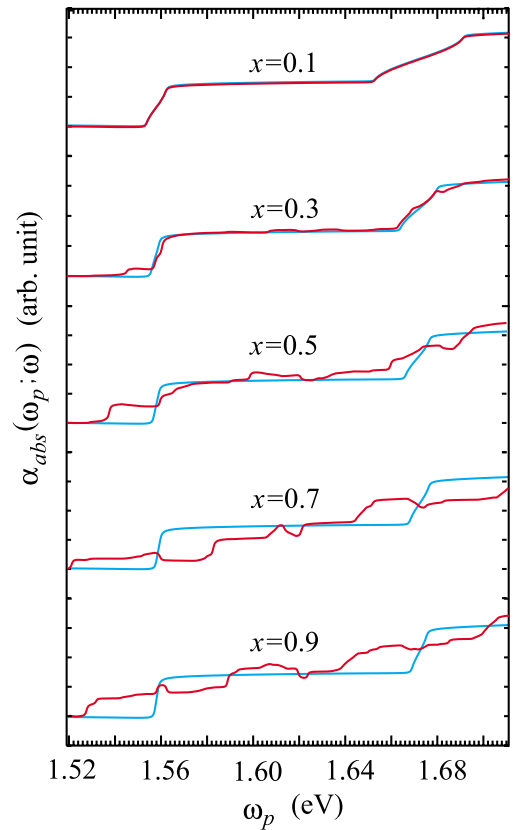

FIG. 5. (Color online) The effective absorption coefficients $\alpha_{a b s}\left(\omega_{p} ; \omega\right)$ as a function of the probe light energy $\omega_{p}$ for the PPT-1 with $x=0.1-0.9$ and $N_{\omega}=3$. The spectra with the ac-ZT are depicted by the red line, while those without it are denoted by the blue line.

ton sidebands with $J \neq 0$, namely, $\chi_{J \neq 0}\left(\omega_{p} ; \omega\right)=0$, because of the absence of the ac-ZT, as easily seen from Eq. (52). In constrast to this, it is noted that the associated quasienergy looks quite complicated due to the repeated contributions from the photon sidebands, as seen in Fig. 3. The similar explanation is also applicable to Fig. 6 for $x=0.1$.

As $x$ increases in Figs. 5 and 6, the profile shows the following anomalous behavior. At $x=0.3$, a number of dimples superimposed on the absorption spectra seen at $x$

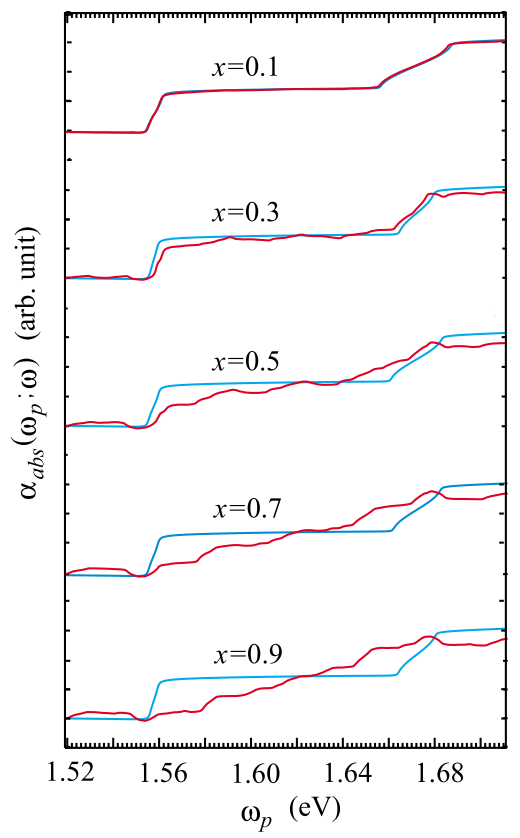

FIG. 6. (Color online) The same as Fig. 5 but for the PPT-2. 
$=0.1$ are found in the vicinity of $\omega_{p}=1.56$ and $1.68 \mathrm{eV}$, and these are modified to conspicuous dents with further increase of $x$. Such anomaly is obviously governed by the ac-ZT, as compared with the reference spectra (the blue curves) without it. Further, a lot of transitions to the photon sidebands, $[(1,1), J \neq 0]$ and $[(2,2), J \neq 0]$, are observed to form the dents in the absorption spectra. These are also due to the ac-ZT. The presence of the anomalous dent structure is the primary finding of this paper.

Finally, it is mentioned how the exciton effect on the IDWSL modifies the absorption spectra discussed above, just from the qualitative viewpoint without implementing actual calculations. Inclusion of this effect would provide more realistic spectra than those of Figs. 5 and 6. By applying the similar formulation developed in Sec. II to the excitonic Hamiltonian provided by adding the electron-electron Coulomb interaction to the Hamiltonian of Eq. (3), the following optical susceptibility is obtained within the approximation of neglecting the effects of the many-body Coulomb exchange and correlation:

$$
\chi_{J}^{(e x)}\left(\omega_{p} ; \omega\right)=\frac{1}{\epsilon_{0}}\left|d_{0}^{(v c)}\right|^{2} \sum_{\bar{\nu}} \frac{\widetilde{\mathcal{O}}_{J \bar{\nu}}^{(e x)}}{E_{\bar{\nu}}^{(e x)}-\omega_{p}-i \gamma}
$$

in place of Eq. (51). Moreover, the excitonic absorption spectra are given by

$$
\alpha_{a b s}^{(e x)}\left(\omega_{p} ; \omega\right)=\frac{\omega_{p}}{c} \sum_{J} \operatorname{Im} \chi_{J}^{(e x)}\left(\omega_{p} ; \omega\right)
$$

in place of Eq. (54). Here, the notations with the superscript (ex) have the meanings corresponding to those without this, and the barred index $\bar{\nu}$ has been used in Eq. (63) to put stress on the difference of this index indicating the excitonic Floquet state from index $\nu$ in Eq. (51), indicating the nonexcitonic Floquet state. It is remarked that neither $k$ nor $\mathbf{K}_{\|}$are good quantum numbers any longer since these Bloch states are mixed together when the electron-electron Coulomb interaction is diagonalized to obtain the exciton quasienergy $E_{\bar{\nu}}^{(e x)}$. The spectra of the excitonic Floquet state $\bar{\nu}$ are composed of the two components ascribable to exciton bound and continuum states. It seems that aside from the factor of the Coulomb enhancement, the spectral profile of the continuum component remains almost the same as the spectral profile shown in Figs. 5 and 6 without the exciton effect. On the other hand, there is no correspondence of the bound-state component in these figures. It is seems that this component manifests itself to a certain extent below the absorption edge of the continuum component belonging to the same manifold specified by, for example, $[B, J]$. Such extent is almost proportional to the degree of magnitude of the exciton binding energy, namely, $\left|E_{\bar{\nu}}^{(e x)}-\left[E_{\nu}(k=0)+\epsilon_{\|}^{(v c)}\left(\mathbf{K}_{\|}=0\right)\right]\right|$. In general, the binding energy is much greater in the manifold of the parent band $(J=0)$ than in that of the photon sideband $(J$ $\neq 0$ ); hence, this energy tends to be reduced with increasing $|J|$. Therefore, it is speculated that the excitonic effective absorption spectra are more or less modified from the spectra of Figs. 5 and 6 around $\omega_{p}=1.56$ and $1.68 \mathrm{eV}$, whereas these remain almost unaltered away from this region.

\section{Differential effective absorption coefficient: $\alpha_{a b s}^{0}\left(\omega_{p} ; \omega\right)$}

In order to point out the anomalous alteration of the spectral profile in $\alpha_{a b s}\left(\omega_{p} ; \omega\right)$ and to develop the discussion of the exciton effect on it, the alternative effective absorption coefficient $\alpha_{a b s}^{0}\left(\omega_{p} ; \omega\right)$ is defined first as follows:

$$
\alpha_{a b s}^{0}\left(\omega_{p} ; \omega\right)=\frac{\omega_{p}}{c} \sum_{J} \operatorname{Im} \chi_{J}^{0}\left(\omega_{p} ; \omega\right),
$$

where

$$
\chi_{J}^{0}\left(\omega_{p} ; \omega\right)=\frac{1}{\epsilon_{0}}\left|d_{0}^{(v c)}\right|^{2} \sum_{\nu, k} \frac{\widetilde{\mathcal{O}}_{J \nu}(k)}{E_{\nu}(k)-\omega_{p}-i \gamma} .
$$

Further, similar to Eq. (50), $\chi^{0}(t)$ is defined as

$$
\chi^{0}(t)=\sum_{J} e^{J \omega t} \chi_{J}^{0}\left(\omega_{p} ; \omega\right) .
$$

$\chi_{J}^{0}\left(\omega_{p} ; \omega\right)$ is connected with $\chi_{J}\left(\omega_{p} ; \omega\right)$ through the relation

$$
\chi_{J}^{0}\left(\omega_{p} ; \omega\right)=\frac{1}{\mathcal{D}_{\|}} \frac{\partial \chi_{J}\left(\omega_{p} ; \omega\right)}{\partial \omega_{p}} .
$$

Using Eqs. (54) and (65), one obtains

$$
\alpha_{a b s}^{0}\left(\omega_{p} ; \omega\right)=\frac{\omega_{p}}{\mathcal{D}_{\|}} \frac{\partial\left[\alpha_{a b s}\left(\omega_{p} ; \omega\right) / \omega_{p}\right]}{\partial \omega_{p}} \approx \frac{1}{\mathcal{D}_{\|}} \frac{\partial \alpha_{a b s}\left(\omega_{p} ; \omega\right)}{\partial \omega_{p}},
$$

where the fact that $\alpha_{a b s}\left(\omega_{p} ; \omega\right) / \omega_{p}$ is negligibly smaller than $\partial \alpha_{a b s}\left(\omega_{p} ; \omega\right) / \partial \omega_{p}$ has been employed in the second equality. Hereafter, $\alpha_{a b s}^{0}\left(\omega_{p} ; \omega\right)$ is termed the differential effective absorption coefficient because of the above proportion relation to the $\alpha_{a b s}\left(\omega_{p} ; \omega\right)$ differential in $\omega_{p}$.

Similar to Sec. III B, the general survey of $\alpha_{a b s}^{0}\left(\omega_{p} ; \omega\right)$ is taken. If the limit of $\gamma \rightarrow 0$ is taken, the imaginary part of Eq. (66) becomes

$$
\operatorname{Im} \chi_{J}^{0}\left(\omega_{p} ; \omega\right) \approx \frac{\pi}{\epsilon_{0}}\left|d_{0}^{(v c)}\right|^{2} \sum_{\nu, k} \operatorname{Re}\left[\widetilde{\mathcal{O}}_{J \nu}(k)\right] \delta\left(E_{\nu}(k)-\omega_{p}\right),
$$

where just the on-the-energy-shell contribution is taken account of, similar to Eq. (61). Further, the one-dimensional density of state, $\mathcal{D}_{\perp}\left(\omega_{p}\right)$, for the electronic motion in the direction of crystal growth is given by

$$
\mathcal{D}_{\perp}\left(\omega_{p}\right)=\sum_{\nu, k} \delta\left[E_{\nu}(k)-\omega_{p}\right] .
$$

This shows the sequence of simple peak structures. By comparing Eq. (70) with this, it is obviously seen that aside from $\operatorname{Re}\left[\widetilde{\mathcal{O}}_{J \nu}(k)\right], \alpha_{a b s}^{0}\left(\omega_{p} ; \omega\right)$ reflects this density of state for the IDWSL Floquet state. Therefore, it is expected that such a peak structure is more or less modified by introducing the ac-ZT. Differing from $\alpha_{a b s}\left(\omega_{p} ; \omega\right)$, however, each profile of the IDWSL-Floquet state in $\alpha_{a b s}^{0}\left(\omega_{p} ; \omega\right)$ does not suffer from superimposition of the accumulated profiles of other states with smaller quasienergies; in other words, the spectral profiles of these Floquet states are separated from each other. In 


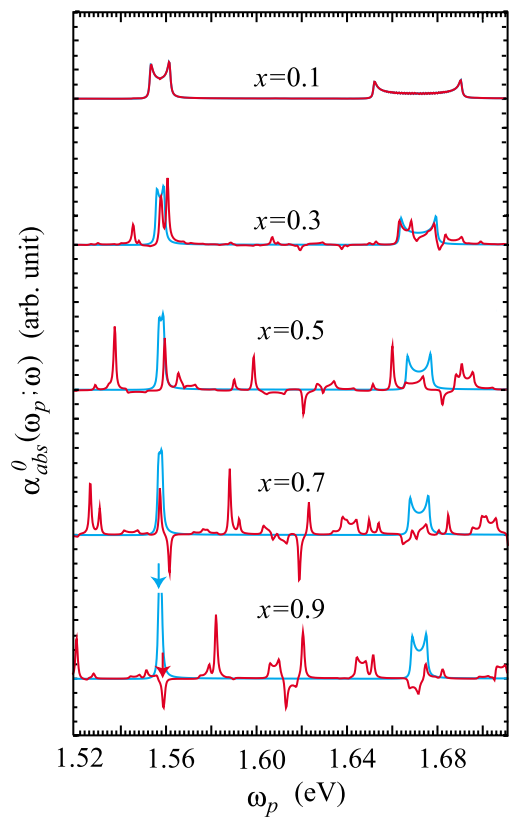

FIG. 7. (Color online) The effective absorption coefficients $\alpha_{a b s}^{0}\left(\omega_{p} ; \omega\right)$ as a function of the probe light energy $\omega_{p}$ for the PPT-1 with $x=0.1-0.9$ and $N_{\omega}=3$. The spectra with the ac-ZT are depicted by the red line, while those without it are denoted by the blue line. The arrowed portions in the spectra for $x=0.9$ are used to aid the discussion later made in Sec. III C. For more details, consult the text.

contrast with this, the examination of the IDWSL-Floquet state in $\alpha_{a b s}\left(\omega_{p} ; \omega\right)$ is more or less hindered from such contamination attributed to the other states. In this sense, $\alpha_{a b s}^{0}\left(\omega_{p} ; \omega\right)$ is regarded as more suitable and straightforward than $\alpha_{a b s}\left(\omega_{p} ; \omega\right)$. This is the reason why $\alpha_{a b s}^{0}\left(\omega_{p} ; \omega\right)$ has been adopted as a more suitable tool than $\alpha_{a b s}\left(\omega_{p} ; \omega\right)$ to explore the IDWSL electronic structure that is the primary purpose of this paper, as mentioned in Sec. I.

The spectra $\alpha_{a b s}^{0}\left(\omega_{p} ; \omega\right)$ are shown in Figs. 7 and 8, which correspond to those in Figs. 5 and 6, respectively. Here, the red and blue curves mean the spectra with and without the ac-ZT, respectively. In Fig. 7, for $x=0.1$, the spectra are almost the same with those of the original SLs with the typical M0 and M1 Van Hove critical points; these singularities are characteristic of $\mathcal{D}_{\perp}\left(\omega_{p}\right)$ for the SLs. As $x$ increases, a greater number of peaks and dips appear in the spectra, and the significant effect of the ac-ZT is clarified since these draw distinction from the reference spectra (the blue curves). In particular, the negative dips stand out for relatively large $x$ 's. A similar discussion also holds in the spectra of Fig. 8. The spectral profiles with negative values are obviously associated with the dent structure seen in Fig. 5. It is notable that the sign and magnitude of the profiles closely reflect those of the correction factor $\operatorname{Re}\left[\widetilde{\mathcal{O}}_{J \nu}(k)\right]$. This shows the advantage of employing the analytical and close expression of Eq. (66) as well as Eq. (51), as is mentioned right below Eq. (52).

Next, the discussion of the excitonic absorption spectra given by Eq. (64) will be developed from the viewpoint of the differential effective absorption coefficient. Here, the ex-

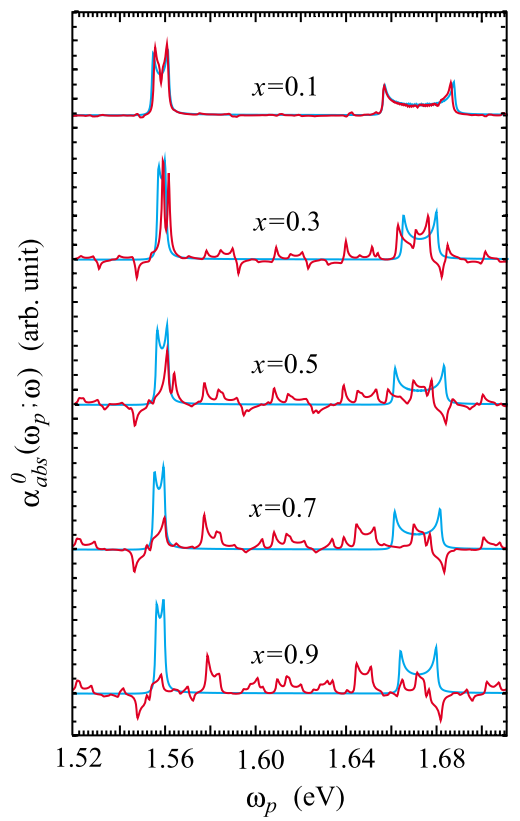

FIG. 8. (Color online) The same as Fig. 7 but for the PPT-2.

citon bound-state component is specifically taken into consideration. It should be noticed again that the following discussion on the exciton effect is just based on the speculation rather than the results obtained by the actual calculations. Let one begin with the expression of Eq. (66). In the case that either the band collapse, namely, the DL, or the band narrowing takes place, $E_{\nu}(k)$ would be able to be replaced, for example, by the quasienergy $E_{\nu}^{0}$ almost independent of $k$. Accordingly, Eq. (66) becomes

$$
\chi_{J}^{0}\left(\omega_{p} ; \omega\right) \approx \frac{1}{\epsilon_{0}}\left|d_{0}^{(v c)}\right|^{2} \sum_{\nu} \frac{\widetilde{\mathcal{O}}_{J \nu}^{0}}{E_{\nu}^{0}-\omega_{p}-i \gamma},
$$

where $\widetilde{\mathcal{O}}_{J \nu}^{0}=\Sigma_{k} \widetilde{\mathcal{O}}_{J \nu}(k)$. It is obviously found that Eq. (63) is comparable to this expression as long as one takes just the discrete sum over $\bar{\nu}$ that includes the bound-state components. Such inferential correspondence between the excitonic Floquet bound state and the nonexcitonic Floquet state with band collapse/narrowing is ascribable to the fact that both states are spatially localized. Actually, Eqs. (72) and (63) provide the spectra with the Lorentzian profiles weighted by $\operatorname{Re}\left[\widetilde{\mathcal{O}}_{J \nu}^{0}\right]$ and $\operatorname{Re}\left[\widetilde{\mathcal{O}}_{J \bar{\nu}}^{(e x)}\right]$, respectively. Therefore, similar to $\alpha_{a b s}^{0}\left(\omega_{p} ; \omega\right)$ of Eq. (65), it would be likely that the negative absorption (the spectral gain) with the dip structure also manifests itself in more realistic exciton spectra $\alpha_{a b s}^{(e x)}\left(\omega_{p} ; \omega\right)$ due to the ac-ZT in the vicinity of $\omega_{p}=E_{\bar{\nu}}^{(e x)}$ if $\operatorname{Re}\left[\widetilde{\mathcal{O}}_{J \bar{\nu}}^{(e x)}\right]$ is negative.

The speculation of the close resemblance between Eqs. (72) and (63) would be ensured in Ref. 13 on the excitonic DWSL driven by the monochromatic and bichromatic lasers though neither dc-ZT nor ac-ZT are taken into account. Here, the spectrum relevant to the bound state with a dominant sharp peak shows a similar profile to the nonexcitonic DLspectrum differential in $\omega_{p}$; for instance, compare Figs. 4(c) 
and 6(c) in Ref. 13. Incidentally, it was illustrated in the usual WSL that the absorption spectra of the excitonic WSL bear a strong resemblance to those of the nonexcitonic WSL differential in $\omega_{p}$, apart from that the peak positions in the former are somewhat shifted to the lower-energy side from the peak positions in the latter by the extent of the binding energies. ${ }^{51}$ Now, let one briefly take account of the arrowed portions in the spectra of Fig. 7 for $x=0.9$. The red and blue arrows indicate the spectra slightly below $\omega_{p}=1.56 \mathrm{eV}$ with and without the ac-ZT, respectively. These portions are associated with the quasienergies that are indicated by the alternative arrows in Figs. 3(c) and 3(f). It is noted that the band narrowing is caused, especially, in the portion of Fig. 3(c) ${ }^{52}$ It is found in Fig. 7 that the strong peak of the spectrum without the ac-ZT (arrow in blue) is drastically changed to the negative absorption with the sharp dip in the spectrum (arrow in red) by incorporating the ac-ZT. Following the above-mentioned speculation of the resemblance between $\alpha_{a b s}^{(e x)}\left(\omega_{p} ; \omega\right)$ and $\alpha_{a b s}^{(0)}\left(\omega_{p} ; \omega\right)$, it would be expected that a similar change of the spectral pattern of the excitonic Floquet bound state would occur in $\alpha_{a b s}^{(e x)}\left(\omega_{p} ; \omega\right)$ as well. The speculation made here will have to be confirmed by the actual calculation of $\alpha_{a b s}^{(e x)}\left(\omega_{p} ; \omega\right)$.

Finally, it should be mentioned that the precedent studies $^{43,44}$ on the coherent dynamics of SLs under a terahertz field are indicative of the existence of the negative absorption in the linear absorption spectra. In these studies, the spectra were obtained by solving the semiconductor Bloch equations within the Hartree-Fock approximation of the many-body Coulomb correlation, but without the ac-ZT in contrast to the present study. Thus, the negative absorption predicted therein is thought to be due to the combination of the exciton and many-body Coulomb exchange effects, differing from the present one that is speculated due to the combination of the exciton and ac-ZT effects.

\section{Origin of the anomaly}

The origin of the negative absorption discussed above is explored by examining $\chi_{J}^{0}\left(\omega_{p} ; \omega\right)$ defined in Eq. (66). It is noticed that $\chi_{J=0}^{0}\left(\omega_{p} ; \omega\right)$ does not contribute to the negative values in $\alpha_{a b s}^{0}\left(\omega_{p} ; \omega\right)$ of Eq. (65), even if the ac-ZT is dominant since

$$
\operatorname{Im} \chi_{0}^{0}\left(\omega_{p} ; \omega\right)=\frac{1}{\epsilon_{0}}\left|d_{0}^{(v c)}\right|^{2} \sum_{\nu, k} \frac{\gamma\left|\mathcal{C}_{0 \nu}(k)\right|^{2}}{\left(E_{\nu}(k)-\omega_{p}\right)^{2}+\gamma^{2}}>0 .
$$

Here, the net number of exchanged photons due to the ac-ZT between Floquet states is zero. Therefore, it is found that the negative absorption is attributed to $\chi_{J}^{0}\left(\omega_{p} ; \omega\right)$ with $J \neq 0$, where the ac-ZT takes place so that the net number of the mediating photons is finite. In order to understand what happens in more detail, the validity of the perturbation expansion with respect to $\mathcal{V}_{[B J],\left[B^{\prime} J^{\prime}\right]}$ of Eq. (39) is assumed. Namely, $\mathcal{C}_{J \nu}(k)$ of Eq. (49) is approximated as

$$
\begin{aligned}
& \mathcal{C}_{J,\left[B_{0} J_{0}\right]}(k) \\
& \quad \approx o_{B_{0}}^{(v c)} \delta_{J J_{0}}+\sum_{B} \frac{o_{B}^{(v c)} \mathcal{V}_{[B J],\left[B_{0} J_{0}\right]}}{\varepsilon_{[B J]}(k)-\varepsilon_{\left[B_{0} J_{0}\right]}(k)} \\
& +\sum_{B B^{\prime} J^{\prime}} \frac{o_{B}^{(v c)} \mathcal{V}_{[B J],\left[B^{\prime} J^{\prime}\right]} \mathcal{V}_{\left[B^{\prime} J^{\prime}\right],\left[B_{0} J_{0}\right]}}{\left.\left[\varepsilon_{[B J]}(k)-\varepsilon_{\left[B_{0} J_{0}\right]}(k)\right]\left[\varepsilon_{\left[B^{\prime} J^{\prime}\right]}(k)-\varepsilon_{\left[B_{0} J_{0}\right]}(k)\right)\right]}+\cdots
\end{aligned}
$$

by using Eq. (38), where the label $\nu$ in view of the ac-ZT has been replaced by the ac-ZT-free label $\left[B_{0}, J_{0}\right]$. The Feynman diagram of $\chi_{J}^{0}\left(\omega_{p} ; \omega\right)$ based on this expansion is depicted in Fig. 9. Here, the vertices of photon absorption and emission associated with the probe laser and the PPT field are denoted by an open circle and a cross, respectively, and the $k$ dependence on $\chi_{J}^{0}\left(\omega_{p} ; \omega\right)$ is suppressed for the sake of simplicity. Further, the propagator $G_{\left[B_{0} J_{0}\right]}\left(\omega_{p}\right) \equiv 1 /\left[\varepsilon_{\left[B_{0} J_{0}\right]}(k)-\omega_{p}-i \gamma\right]$, pertinent to the interband transition by photoabsorption, is represented by a thick vertical line, and the propagator $G_{[B J],\left[B_{0} J_{0}\right]}^{(Z T)} \equiv 1 /\left[\varepsilon_{[B J]}(k)-\varepsilon_{\left[B_{0} J_{0}\right]}(k)\right]$, between the Floquet states $[B, J]$ and $\left[B_{0}, J_{0}\right]$ due to the ac-ZT, is represented by a thin vertical line, where time evolves upward. In addition, a photon line of the PPT field is denoted by a wiggly line. For instance, the second diagram in the second equality of Fig. 9 shows the process that the interband photoabsorption to the state $\left[B_{0}, 0\right]$ is caused by the probe laser to begin with, followed by the ac-ZT from it to the state $[B,-J]$ with accompanying photoemission with $J \omega$, and ending up with the interband photoemission back to vacuum. In Fig. 9, aside from the first diagram, each diagram represents the mediation of $J$-photon emission, where the number of $J$ is the difference of the total number of the emitted photons $\left(J_{\text {emission }}\right)$ from that of the absorbed photons $\left(J_{\text {absorption }}\right)$, namely, $J=J_{\text {emission }}$ $-J_{\text {absorption. }}$ For instance, in both the fourth and sixth diagrams, $J_{\text {emission }}=J+J^{\prime}$ and $J_{\text {absorption }}=J^{\prime}$, while in the fifth diagram, $J_{\text {emission }}=J+J_{0}$ and $J_{\text {absorption }}=J_{0}$.

Next, the dual-time optical susceptibility $\mathcal{X}^{0}\left(t, t^{\prime}\right)$ defined as

$$
\mathcal{X}^{0}\left(t, t^{\prime}\right)=-i \frac{\left(t-t^{\prime}\right)}{\mathcal{D}_{\|}} \mathcal{X}\left(t, t^{\prime}\right),
$$

is discussed to understand the anomaly of $\chi^{0}(t)$ from a different aspect. Here, the dual-time optical susceptibility $\mathcal{X}\left(t, t^{\prime}\right)$ is defined in Eq. (B1), and through a relation similar to Eq. (B3), $\mathcal{X}^{0}\left(t, t^{\prime}\right)$ corresponds to $\chi^{0}(t)$ of Eq. (67). Using Eqs. (B6) and (47), $\mathcal{X}^{0}\left(t, t^{\prime}\right)$ becomes of the form

$$
\mathcal{X}^{0}\left(t, t^{\prime}\right)=-i \Theta\left(t-t^{\prime}\right) \frac{1}{\epsilon_{0}}\left|d_{0}^{(\nu c)}\right|^{2} \sum_{\nu, k, J} e^{-i\left[E_{\nu}(k)-i \gamma\right]\left(t-t^{\prime}\right)} e^{i J \omega t} \widetilde{\mathcal{O}}_{\nu}(k) .
$$

This optical susceptibility keeps track of the time evolution of Floquet states of the IDWSL between $t^{\prime}$ and $t$, at which the interband absorption and emission due to the probe light are caused, respectively, as specified by the vertices (the open circles) in Fig. 9. Although these states are regarded as quasistationary, they are actually "time-dependent and tran- 


$$
\chi_{J}^{0=} \sum_{v}
$$

FIG. 9. The Feynman diagrams indicating the perturbation expansion of $\chi_{J}^{0}\left(\omega_{p} ; \omega\right)$ with respect to the ac-ZT interaction $\mathcal{V}_{[B J],\left[B^{\prime} J^{\prime}\right]}$. Here, time evolves upward. In the first equality, the diagram shows the time evolution based on Eq. (66), where the large filled circles represent $\mathcal{C}_{-J \nu}(k)$ and $\mathcal{C}_{0 \nu}^{*}(k)$, the thick vertical line represents the propagator for the Floquet state $\nu$ given by $1 /\left[E_{\nu}(k)-\omega_{p}-i \gamma\right]$, the small open circles represent the vertices $d_{0}^{(v c)}$ and $d_{0}^{(v c)^{*}}$ due to the probe photon emission and absorption, respectively, and the wiggly line represents $J$-photon emission due to the ac ZT. In the second equality, the cross represents the vertex of $\mathcal{V}_{[B J],\left[B^{\prime} J^{\prime}\right]}$, and the thick and thin vertical lines represent the propagators $G_{\left[B_{0} J_{0}\right]}\left(\omega_{p}\right)$ and $G_{[B J],\left[B_{0} J_{0}\right]}^{(Z T)}\left(\omega_{p}\right)$, respectively. The labels put on the propagators, such as $\left[B^{\prime}, J^{\prime}\right]$, show the mediating ZT-free Floquet states. For more details, consult the text.

sient". Hence, during this temporal interval, the dynamic process of the ac-ZT takes place, giving rise to a transition from one Floquet state to another by emitting/absorbing the net number of photons with $J \omega(J \neq 0)$ at $t\left(>t^{\prime}\right)$. This character differs a great deal from the usual linear optical susceptibility for "stationary" photoexcited states. Moreover, this difference is also seen in the way of the time dependence of $\mathcal{X}^{0}\left(t, t^{\prime}\right)$ from that of the corresponding usual-optical susceptibility. The present linear optical susceptibility is not the function of the time difference, $t-t^{\prime}$, namely, $\mathcal{X}^{0}\left(t, t^{\prime}\right) \not \equiv \mathcal{X}^{0}\left(t-t^{\prime}\right)$, due to the contributions of nonzero $J$ 's, as obviously seen in Eq. (76), whereas the usual one is always its function. ${ }^{53}$
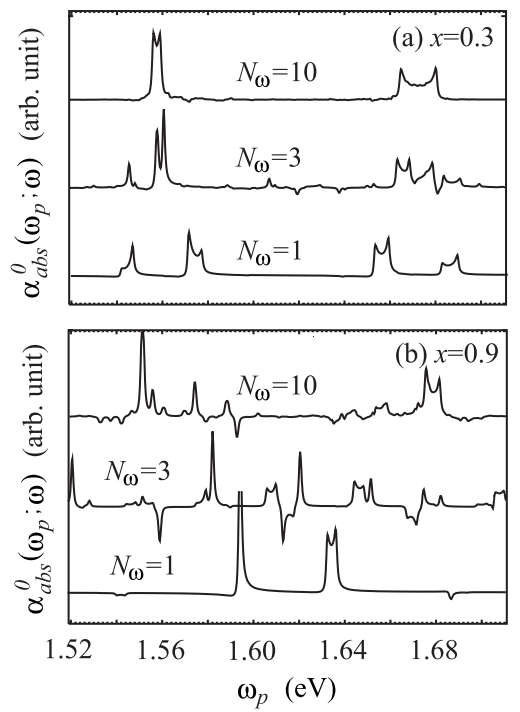

FIG. 10. The $N_{\omega}$ dependence on the effective absorption coefficient $\alpha_{a b s}^{0}\left(\omega_{p} ; \omega\right)$ for the PPT- 1 with $N_{\omega}=1,3$, and 10 , where panels (a) and (b) are for $x=0.3$ and 0.9 , respectively.

\section{E. $N_{\omega}$ and $Z$ dependence}

This subsection is devoted to the miscellany supplemented to the spectral properties of $\alpha_{a b s}^{0}\left(\omega_{p} ; \omega\right)$. Figure 10 shows the $N_{\omega}$ dependence of the spectra (with $Z=10$ ) for the IDWSL driven by the PPT- 1 with $x=0.3$ [Fig. 10(a)] and $x$ $=0.9$ [Fig. 10(b)], where $N_{\omega}$ has been set equal to 1,3 , and 10 , corresponding to $\omega=0.093,0.031$, and $0.01 \mathrm{eV}$, respectively. It is noted again that the ZT-free Floquet state $\left[(2,1),-N_{\omega}\right]$ becomes resonant with that of $[(1,1), 0]$, and so does the ZT-free Floquet state $\left[(1,2), N_{\omega}\right]$ with that of $[(2,2), 0]$. Such $N_{\omega}$-photon resonance is considered stronger with decreasing $N_{\omega}$ through the ac-ZT. This is evident in Fig. $10(\mathrm{a})$, where the effect of the ac-ZT is not completely removed by the PPT- 1 though relatively weak because of the small $x$. For $N_{\omega}=10$, the ac-ZT is suppressed to a large extent, and the resulting spectra are almost identical to the reference spectra without the ac-ZT depicted in Fig. 7 for $x$ $=0.3$ (denoted by a blue curve). The spectra are more modulated for $N_{\omega}=3$ and 1 . In particular, for $N_{\omega}=1$, it is observed that the parent bands of $[(1,1), 0]$ (situated around $\omega_{p}$ $\approx \epsilon_{0(1,1)}^{(v c)}=1.557 \mathrm{eV}$ ) and $[(2,2), 0]$ (situated around $\epsilon_{0(2,2)}^{(v c)}$ $=1.672 \mathrm{eV}$ ) are split into the doublet peaks due to the anticrossings caused by the resonant coupling with the photon sidebands of $\left[(2,1),-N_{\omega}\right]$ and $\left[(1,2), N_{\omega}\right]$, respectively. On the other hand, in Fig. 10(b), the spectral modulation with respect to $N_{\omega}$ is more conspicuous due to the greater effect of the ac-ZT than that in Fig. 10(a). For $N_{\omega}=10$ and 3, the complicated photon sideband structure appears between $\epsilon_{0(1,1)}^{(v c)}$ and $\epsilon_{0(2,2)}^{(v c)}$. Further, the absorption edge, the position of which is $1.553 \mathrm{eV}$ for the original SLs, is greatly redshifted, especially, for $N_{\omega}=3$. As regards $N_{\omega}=1$, the spectral pattern looks much different from the corresponding one in Fig. 10(a). Although the doublet structure is still discernible, one lobe is converted to a faint dip, and the other becomes a stronger peak. Here, the split energy is also greater than that 


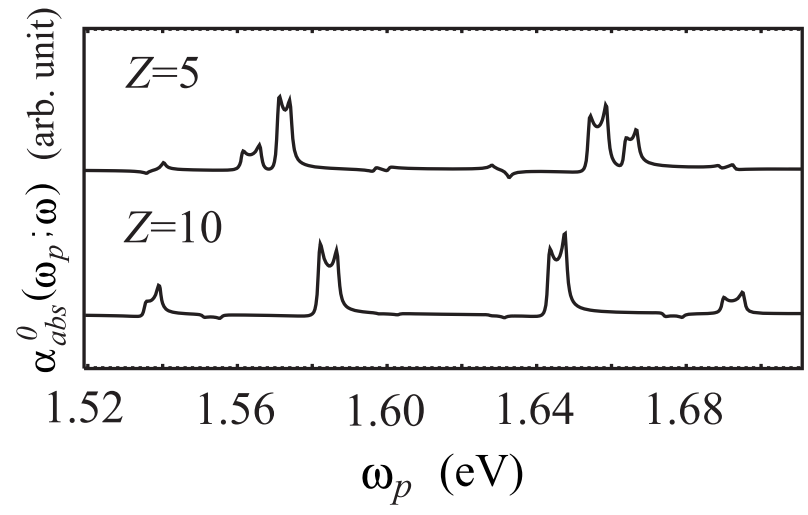

FIG. 11. The $Z$ dependence on the effective absorption coefficient $\alpha_{a b s}^{0}\left(\omega_{p} ; \omega\right)$ for the PPT- 1 with $Z=5$ and 10 , where $N_{\omega}=1$ and $x=0.4$.

in Fig. 10(a), which reflects the stronger anticrossing.

Figure 11 shows the $Z$ dependence of the spectra (with $N_{\omega}=1$ and $x=0.4$ ) for the IDWSL driven by the PPT- 1 with $Z=5$ and 10 . In both spectra, there appear the doublet structures mentioned above. It is seen that the split energy of each doublet becomes reduced with decreasing $Z$, as expected. Therefore, it is evident that the manifestation of the doublet originates from the ac-ZT since this effect is more dominant with greater $Z$ as well as smaller $N_{\omega}$.

\section{SUMMARY AND CONCLUSION}

The joint-miniband DWSL, driven by the PPT-1 and PPT-2 with $F_{d c}^{\prime}=0$, is examined by taking the interminiband interactions due to the ac-ZT into account. In order to understand the quasienergy structure of the IDWSL Floquet state and its modulation pattern by the tuning of these PPTs, the linear interband absorption spectra $\alpha_{a b s}\left(\omega_{p} ; \omega\right)$ of this IDWSL caused by the weak probe light are examined based on the semiconductor Bloch equations. With decreasing $x$ in these PPTs, the unexpected dent structures manifest themselves more conspicuously in $\alpha_{a b s}\left(\omega_{p} ; \omega\right)$ as a function of $\omega_{p}$. These profiles are much different from those for $x \approx 0$ showing the sequence of the ascending steplike structures similar to the original SLs simply due to the coherent removal of the ac-ZT by the PPTs. Moreover, the alternative absorption spectra $\alpha_{a b s}^{0}\left(\omega_{p} ; \omega\right) \propto \partial \alpha_{a b s}\left(\omega_{p} ; \omega\right) / \partial \omega_{p}$ are also evaluated to stress the variation of $\alpha_{a b s}\left(\omega_{p} ; \omega\right)$ as a function of $\omega_{p}$ and to obtain deeper insight into the IDWSL. The anomalous dent structures become spectral dips showing the negative absorption in $\alpha_{a b s}^{0}\left(\omega_{p} ; \omega\right)$. According to the mathematical analysis and the Feynman-diagrammatic consideration of Eq. (66), it is seen that this anomaly is attributed to the nonlinear optical interminiband transitions accompanying the nonzero net energy exchange, $J \omega(J \neq 0)$, due to the ac-ZT. This anomalous behavior is also reflected in the unusual time dependence of the dual-time optical susceptibility $\mathcal{X}^{0}\left(t, t^{\prime}\right)$. That is, this is not the function of the difference between the time $t^{\prime}$ of the outset of the interaction with the probe light and the time $t$ of the response to this interaction, namely, $t-t^{\prime}$, since the nonzero net number of photons with $J \omega$ are emitted/absorbed during the interval of $t^{\prime}$ and $t$. In addition, the more realistic absorption spectra $\alpha_{a b s}^{(e x)}\left(\omega_{p} ; \omega\right)$ including the exciton effect is mentioned, but without actual calculations. Based on the close resemblance between $\alpha_{a b s}^{(e x)}\left(\omega_{p} ; \omega\right)$ and the spectral portion of $\alpha_{a b s}^{0}\left(\omega_{p} ; \omega\right)$ showing the quasienergy-band narrowing and collapse, it is speculated that the negative absorption likely occurs in the excitonic spectra.

The findings obtained in this paper are considered significant as the first characterization of the IDWSL spectra. However, the more sophisticated calculations in view of the exciton effect and the electron-electron correlation are needed, in particular, to confirm the presence of the negative absorption in more realistic absorption spectra comparable to experiments and the other theoretical studies. ${ }^{43,44}$ The calculations toward this direction is now in progress. Moreover, it is remarked that the negative absorption of concern here is implied to be realized without population inversion of the SL system; although the well-known lasing without population inversion in the driven atomic lambda system ${ }^{54}$ has been investigated intensively thus far, this is attributed to the different origin from the present one.

\section{ACKNOWLEDGMENT}

This research was financially supported by Grant-in-Aid for Scientific Research (B) and Grant-in-Aid for Scientific Research (C) from the Japan Society for the Promotion of Science.

\section{APPENDIX A: SUMMARY OF THE ADOPTED APPROXIMATIONS AND CRITERIA OF THE VALIDITY}

Several approximations and assumptions are made in the text, though not verified thus far. The purpose of this appendix is to enumerate them again and to show the criteria of the validity for the adopted approximations with higher importance. First, the approximations and assumptions employed here are listed in the descending order of importance, with brief explanations, if necessary.

(i) The NNTB model is employed throughout this paper [see Eqs. (4) and (12)].

(ii) $Z_{\lambda \lambda^{\prime}}^{(s)}$ of Eq. (13), given by

$$
Z_{\lambda \lambda^{\prime}}^{(s)}=\int d z\langle\lambda \mid z\rangle z\left\langle z \mid \lambda^{\prime}\right\rangle,
$$

is evaluated by using the IPBQW model. By approximating the Wannier function, $\langle z \mid \lambda\rangle=w_{b}(z-l d)$, by a corresponding wave function given by the IPBQW model, one obtains

$$
Z_{\lambda \lambda^{\prime}}^{(s)}=l d \delta_{\lambda \lambda^{\prime}}+X_{b b^{\prime}}^{(s)} \delta_{l l^{\prime}}\left(1-\delta_{b b^{\prime}}\right)
$$

where the first term, $l d$, and the second term, given by 


$$
X_{b b^{\prime}}^{(s)}= \begin{cases}\frac{2}{\pi^{2}}(-1)^{b+b^{\prime}-1 / 2}\left[-\frac{1}{\left(b+b^{\prime}\right)^{2}}+\frac{1}{\left(b-b^{\prime}\right)^{2}}\right] d & \text { (if } b+b^{\prime}-1 \text { is even) } \\ 0 & \text { (if } b+b^{\prime}-1 \text { is odd) }\end{cases}
$$

are associated with the intraminiband and interminiband transitions, respectively. According to Eq. (A2), oblique transitions between different l's are neglected, namely, $Z_{(b, l)\left(b^{\prime}, l^{\prime} \neq l\right)}^{(s)}=0$. In addition, $X_{12}^{(s)}=-16 d / 9 \pi^{2}$.

(iii) $\mathcal{F}_{[B J],\left[B^{\prime} J^{\prime}\right]}$ of Eq. (40) is evaluated by use of the highfrequency approximation. ${ }^{16}$ This is equivalent to the replacement of the exponent of Eq. (35), $\varepsilon_{[B J]}(k)-\int^{t} d t^{\prime} \epsilon_{B}\left(k-\frac{A\left(t^{\prime}\right)}{c}\right)$, by $J \omega t$.

(iv) In the formulation of Sec. II, it is assumed that the Coulomb interactions for the exciton effect and the electron correlation effect are neglected.

(v) In Eq. (54), the approximation $D(n, J) \approx \delta_{n 0}$ has been made by assuming that $\Delta \ll 1$ holds correctly.

(vi) Because of $F_{a c} \gg f_{p 0}$ and $\omega \ll \omega_{p}$, it is assumed that $F(t)$ does not contribute to the interband transitions and $f_{p}(t)$ does not contribute to the intersubband transitions [see Eqs. (4)-(6)]. Further, in Eq. (46), it is assumed that the degree of magnitude of $P(t)$ is negligibly smaller than $\epsilon_{0} f_{p 0}$.

(vii) In the formulation of Sec. II, only a single electronhole pair is considered.

Next, the validity of the items (i), (ii), and (v) is mainly taken into account, and the criteria of the applicability of these approximations are indicated. To begin with, let item (i) be considered. Figure 12 shows the lowest three miniband energies of the electrons $(b=1-3)$ in the $c$ band [panel (a)] and the $v$ band [panel (b)] for the SLs of concern in the present paper, namely, 35ML-GaAs/ $11 \mathrm{ML}-\mathrm{Ga}_{0.75} \mathrm{Al}_{0.25} \mathrm{As}$. The solid curves represent the results obtained by the fully numerical calculations, which is cited from Fig. 1 of Ref. 49, while the dashed curves show the energies given by the NNTB model of Eq. (32), where the miniband widths employed are fitted to those given in Fig. 1 of Ref. 49. By comparing these two curves, it is seen that the NNTB model works well, especially, in the lowest miniband of the $c$ band and the lowest three minibands of the $v$ band. In the text, the lowest two minibands of the $c$ and $v$ bands are adopted for further calculations (see Sec. III). It is noted that the resulting criterion of the validity for the NNTB model would likely break down in other SL systems composed of shallower QWs with a narrower barrier potential.

As regards item (ii), Fig. 13 showing the quasienergies of an electron in the $c$ band would be able to provide the criterion of the validity for the IPBQW approximation. The system of concern here is the SLs of $35 \mathrm{ML}-\mathrm{GaAs} / 11 \mathrm{ML}-\mathrm{Ga}_{0.75} \mathrm{Al}_{0.25} \mathrm{As}$ (Table I) under the monochromatic ac field $(x=\mu=1, \delta=0)$ with $F_{a c}$ $=0-300 \mathrm{kV} / \mathrm{cm}$ and $\omega=\Omega_{\text {Bloch }}$ and the dc field with $F_{d c}^{\prime}$ $=F_{d c}=24 \mathrm{kV} / \mathrm{cm}$. Note that the dc-ZT plays an important role in this system since the resonant dc-ZT across three barriers takes place, ${ }^{21}$ this situation corresponds to the case of $N_{\omega}=3$. Although the case of $F_{d c}^{\prime}=0$ causing no dc-ZT is exclusively taken into account in the text, the case of $F_{d c}^{\prime}$ $\neq 0$ is also discussed here so that the criterion of the validity for the IPBQW model is comprehensively clarified. As mentioned above, $\hat{Z}_{\lambda \lambda^{\prime}}^{(s)}$ of Eq. (13) is relevant to dipole interactions caused by the dc and ac fields, accompanying the intraminiband transition and the interminiband transition, where the intraminiband interaction due to the ac field is associated with the photon-assisted tunneling and the interminiband interactions due to the $\mathrm{dc}$ and ac fields correspond

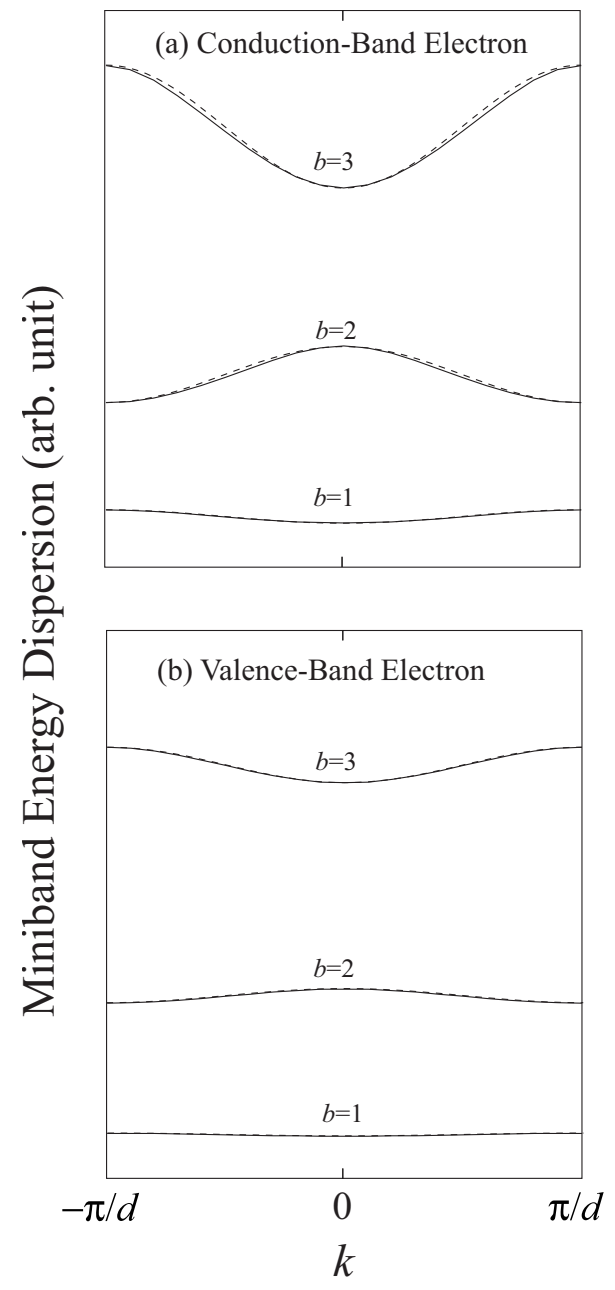

FIG. 12. The lowest three miniband energies of the electrons $(b=1-3)$ as a function of the Bloch momentum $k$ in the $c$ band [panel (a)] and the $v$ band [panel (b)] for the SLs of $35 \mathrm{ML} \mathrm{GaAs} / 11$ ML $\mathrm{Ga}_{0.75} \mathrm{Al}_{0.25} \mathrm{As}$. The solid curves represent the results obtained by the fully numerical calculations, while the dashed curves show the results given by the NNTB model of Eq. (32). For more details, consult the text. 


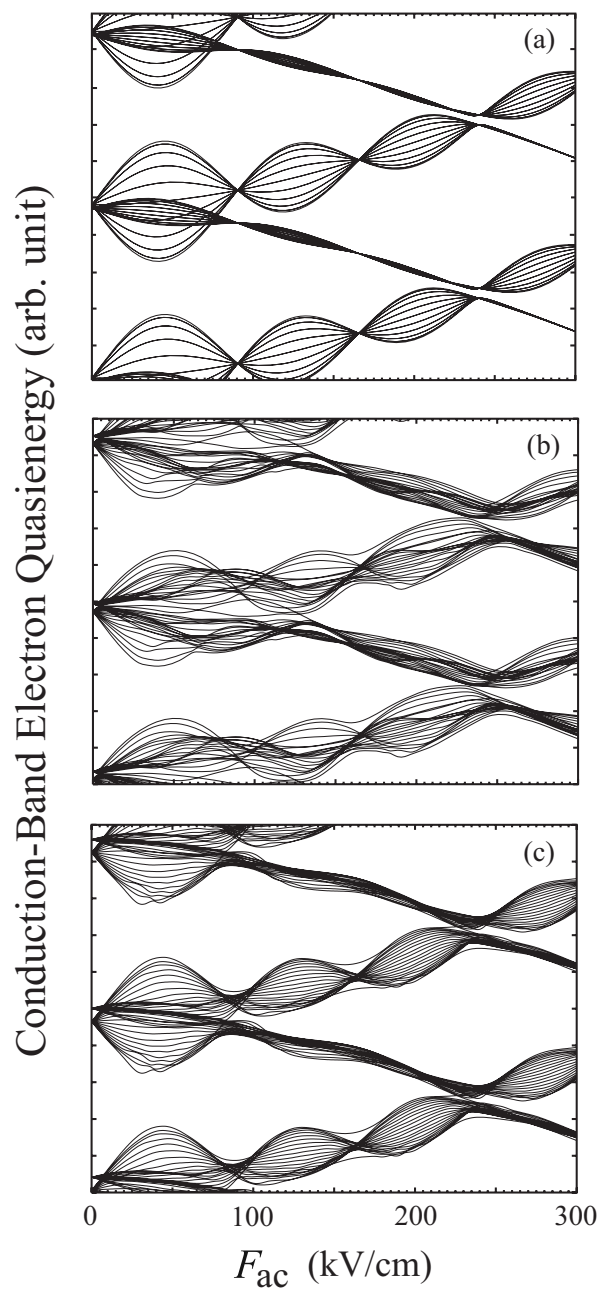

FIG. 13. The DWSL quasienergy of the $c$-band electron as a function of $F_{a c}$ in the system of the SLs of $35 \mathrm{ML} \mathrm{GaAs} / 11 \mathrm{ML}$ $\mathrm{Ga}_{0.75} \mathrm{Al}_{0.25} \mathrm{As}$ exposed to the monochromatic ac field and the $\mathrm{dc}$ field with $F_{d c}^{\prime}=F_{d c}=24 \mathrm{kV} / \mathrm{cm}$. The results in panels (a), (b), and (c) are obtained by the IPBQW model, the fully numerical calculations with both dc- and ac-ZTs, and the fully numerical calculations with just the ac-ZT, respectively. The results of (b) and (c) are cited from Ref. 21. For more details, consult the text.

to the dc- and ac-ZTs, respectively. The result of Fig. 13(a) is obtained based on the IPBQW approximation of Eq. (A2) by solving the eigenvalue equation given in Eq. (10) of Ref. 35, similar to Eq. (38) without contributions from the $v$ band. On the other hand, the results of Figs. 13(b) and 13(c) are cited from Figs. 4(i) 4(ii) of Ref. 21, respectively, where the quasienergy shown in Fig. 13(b) is obtained by the fully numerical calculations with both dc- and ac-ZTs, while that of Fig. 13(c) is obtained by neglecting the dc-ZT from the above calculation for Fig. 13(b).

By comparing the quasienergy of Fig. 13(a) with the quasienergies of Figs. 13(b) and 13(c), the degree of inaccuracy arising from the IPBQW model would be evaluated. In spite of the fact that there is an overall similarity between the quasienergies shown in Figs. 13(a) and 13(b) regarding the positions of the band narrowing and of the anticrossing between different photon sidebands, it seems that the IPBQW model fails to reproduce the quasienergy in further detail, in particular, in the lower- $F_{a c}$ region where the variation pattern of the quasienergy is much entangled in Fig. 13(b). However, by comparing Fig. 13(a) with Fig. 13(c), it is found that the agreement between them is fairly improved. This observation would be interpreted by resorting to the perturbation theory for a degenerate dressed QW system, where its energy is labeled by $(b, l, j)$, with $b$ and $l$ being the indices of a miniband and a QW site as before and $j$ being the number of photon sidebands. Herein, let the three dressed QW states labeled as $(b=1, l, j),\left(b^{\prime}=2, l^{\prime} \equiv l+\Delta l, j\right)$, and $\left(b^{\prime}=2, l, j^{\prime}\right.$ $\equiv j+\Delta j$ ) be termed S1, S2, and S3, respectively, for the sake of simplicity. Due to the resonant dc-ZT in the case concerned, $\mathrm{S} 1$ is resonantly coupled to $\mathrm{S} 2$ across three barriers, namely, $|\Delta l|=N_{\omega}=3$. Therefore, Eq. (A2) resulting from the IPBQW model cannot account for the dc-ZT correctly because of $\Delta l \neq 0$. On the other hand, due to the ac-ZT, S1 is also resonantly coupled to S3 by absorbing/emitting three photons, namely, $|\Delta j|=3$. Since $V_{\Delta j}$ of Eq. (42) is read as $V_{\Delta j} \propto \delta_{\Delta j, 1}+\delta_{\Delta j,-1}$ in this case of the monochromatic laser driving, the leading contribution of the ac-ZT arises from third-order perturbation terms with respect to $V_{\Delta j}$. According to the above discussion, the result of Fig. 13(a) does not incorporate the effect of the dc-ZT at all, and the prominent difference from that of Fig. 13(b) is attributed to the lack of this effect. This difference is reduced, however, in the region of $F_{a c} \gg F_{d c}$. The degree of accuracy of the approximate expression of Eq. (A2) for evaluating the ac-ZT is assessed by comparing Fig. 13(a) with Fig. 13(c). It is found that there is just a slight difference between them over the entire $F_{a c}$ region. To conclude, the IPBQW model is ensured in the system concerned in the text since only the case of $F_{d c}^{\prime}=0$ is focused (see Sec. III). In addition, it is speculated that this model is also safely applied to the case of $F_{d c}^{\prime} \neq 0$ if this field gives rise to just weak and nonresonant dc-ZT.

Relevant to item (ii), the following two supplemental remarks are made. The first is regarding the fact that both the NNTB model and the high-frequency approximation, pertinent to items (i) and (iii), respectively, are incorporated as well in the calculation for Fig. 13(a), However, it would be considered that the numerical inaccuracy due to both of these approximations is limited since the validity of the NNTB has already been confirmed, as mentioned above, and that of the high-frequency approximation is also confirmed in Ref. 16 in the case of a monochromatic ac-field driving. Incidentally, in fact, in the case of the driving by the PPT-2 with the squarewave form, the high-frequency approximation applied to $\mathcal{F}_{[B J],\left[B^{\prime} J^{\prime}\right]}$ of Eq. (40) gives an exact result, though not shown here. The second remark is regarding the ZT-free Hamiltonian of Eq. (34) based on the IPBQW approximation. This model has been widely used as the ansatz to account for the WSL and ZT-free DWSL. However, the application of this should be limited to the SL systems with relatively wide and high potential barriers since $\hat{Z}_{0}^{(s)}$ is provided by the IPBQW approximation.

Finally, the approximation relevant to item (v) is confirmed. Figure 14 shows the spectra of $\alpha_{a b s}^{0}\left(\omega_{p} ; \omega\right)$ given by replacing $\chi_{J}\left(\omega_{p}, \omega\right)$ of Eqs. (53) and (54) by $\chi_{J}^{0}\left(\omega_{p}, \omega\right)$ of Eq. (66) for the IDWSL driven by the PPT- 1 with $x=0.6$. The red 


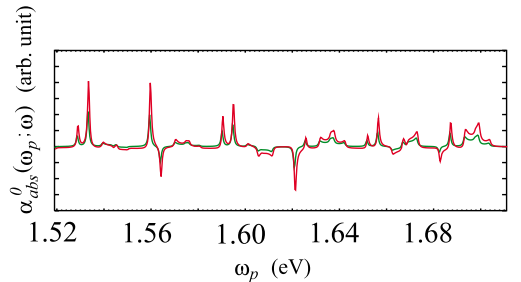

FIG. 14. (Color online) The $\Delta$ dependence on the effective absorption coefficient $\alpha_{a b s}^{0}\left(\omega_{p} ; \omega\right)$ for the PPT-1 with $x=0.6$ and $N_{\omega}$ $=3$. In the red and green traces, the $\Delta$ dependence is included and excluded, respectively. For more details, consult the text.

and green traces are obtained by using Eqs. (53) and (54), respectively. It is seen that there is little difference between the two. Therefore, $\alpha_{a b s}^{0}\left(\omega_{p} ; \omega\right)$ is correctly approximated by Eq. (65) and is related to $\alpha_{a b s}\left(\omega_{p} ; \omega\right)$ through Eq. (69).

\section{APPENDIX B: DUAL-TIME OPTICAL SUSCEPTIBILITY}

The relation of $\chi(t)$ defined in Eq. (20) with the dual-time optical susceptibility $\mathcal{X}\left(t, t^{\prime}\right)$, defined in general as

$$
P(t)=\epsilon_{0} \int_{-\infty}^{t} d t^{\prime} \mathcal{X}\left(t, t^{\prime}\right) f_{p}^{(+)}\left(t^{\prime}\right)+(\text { c.c })
$$

is taken into account for the purpose of supplementing the discussion of Sec. III D. By rewriting $\mathcal{X}\left(t, t^{\prime}\right)$ as

$$
\mathcal{X}\left(t, t^{\prime}\right)=\frac{1}{2 \pi} \int_{-\infty}^{\infty} d w e^{i w t^{\prime}} \tilde{\mathcal{X}}(t, w),
$$

and putting it into Eq. (B1) followed by comparison with Eq. (20), $\chi(t)$ is given by

$$
\chi(t)=\lim _{\delta \rightarrow+0} \tilde{\mathcal{X}}\left(t, \omega_{p}+i \delta\right) e^{i\left(\omega_{p}+i \delta\right) t}
$$

owing to the Cauchy integral formula if $\tilde{\mathcal{X}}(t, w)$ has no poles in the upper-half complex $w$ plane. This condition is immediately confirmed by comparing Eq. (B3) with Eq. (21). It yields the explicit form of $\tilde{\mathcal{X}}(t, w)$ as follows:

$$
\tilde{\mathcal{X}}(t, w)=\frac{1}{\epsilon_{0}}\left|d_{0}^{(v c)}\right|^{2} \lim _{\delta \rightarrow+0} \sum_{\nu, \mathbf{K}_{\|}, k} \frac{e^{-i w t} \mathcal{O}_{\nu}(k, t)}{E_{\nu}+\epsilon_{\|}^{(v c)}\left(\mathbf{K}_{\|}\right)-w-i \gamma^{\prime}},
$$

showing that all poles are located at $w=E_{\nu}+\epsilon_{\|}^{(v c)}\left(\mathbf{K}_{\|}\right)-i \gamma^{\prime}$ in the lower-half plane because of $\gamma^{\prime}=\gamma-\delta>0$. Moreover, by putting Eq. (B4) back into Eq. (B2), one obtains

$$
\begin{aligned}
\mathcal{X}\left(t, t^{\prime}\right)= & -i \Theta(t \\
& \left.-t^{\prime}\right) \frac{1}{\epsilon_{0}}\left|d_{0}^{(v c)}\right|^{2} \sum_{\nu, \mathbf{K}_{\|, k}} e^{-i\left[E_{\nu}+\epsilon_{\|}^{(v c)}\left(\mathbf{K}_{\|}\right)-i \gamma\right]\left(t-t^{\prime}\right)} \mathcal{O}_{\nu}(k, t) \\
= & \frac{\mathcal{D}_{\|} \Theta\left(t-t^{\prime}\right)}{\left(t-t^{\prime}\right)} \frac{1}{\epsilon_{0}}\left|d_{0}^{(v c)}\right|^{2} \sum_{\nu, k} e^{-i\left[E_{\nu}-i \gamma\right]\left(t-t^{\prime}\right)} \mathcal{O}_{\nu}(k, t) .
\end{aligned}
$$

\footnotetext{
*Also at Center for Computational Sciences, University of Tsukuba, 1-1-1 Tennodai, Tsukuba, Ibaraki 305-8577, Japan. †hino@bk.tsukuba.ac.jp

${ }^{1}$ S. Haroche, C. Cohen-Tannoudji, C. Audoin, and J. P. Schermann, Phys. Rev. Lett. 24, 861 (1970).

${ }^{2}$ G. Xu and D. J. Heinzen, Phys. Rev. A 59, R922 (1999).

${ }^{3}$ I. Bialynicki-Birula, M. Kaliński, and J. H. Eberly, Phys. Rev. Lett. 73, 1777 (1994).

${ }^{4}$ A. F. Brunello, T. Uzer, and D. Farrelly, Phys. Rev. Lett. 76, 2874 (1996).

${ }^{5}$ F. Grossmann, T. Dittrich, P. Jung, and P. Hänggi, Phys. Rev. Lett. 67, 516 (1991).

${ }^{6}$ M. Grifoni and P. Hänggi, Phys. Rep. 304, 229 (1998).

${ }^{7}$ L. Esaki and R. Tsu, IBM J. Res. Dev. 14, 61 (1970).

${ }^{8}$ K. W. Madison, M. C. Fischer, and M. G. Raizen, Phys. Rev. A 60, R1767 (1999).

${ }^{9}$ D. H. Dunlap and V. M. Kenkre, Phys. Rev. B 34, 3625 (1986).

${ }^{10}$ M. Holthaus, Phys. Rev. Lett. 69, 351 (1992).

${ }^{11}$ J. Zak, Phys. Rev. Lett. 71, 2623 (1993).

${ }^{12}$ T. Meier, F. Rossi, P. Thomas, and S. W. Koch, Phys. Rev. Lett. 75, 2558 (1995).

${ }^{13}$ K. Yashima, K. I. Hino, and N. Toshima, Phys. Rev. B 68, 235325 (2003).

${ }^{14}$ Z.-G. Wang, D. Suqing, and X.-G. Zhao, Phys. Lett. A 353, 210 (2006).

${ }^{15}$ M. Holthaus, G. H. Ristow, and D. W. Hone, Phys. Rev. Lett. 75, 3914 (1995).

${ }^{16}$ M. Holthaus and D. W. Hone, Philos. Mag. B 74, 105 (1996).

${ }^{17}$ K. A. Pronin and A. D. Bandrauk, Phys. Rev. B 69, 195308 (2004).

${ }^{18}$ K. A. Pronin, P. Reinecker, and A. D. Bandrauk, Phys. Rev. B 71, 195311 (2005).

${ }^{19}$ J. Rotvig, A.-P. Jauho, and H. Smith, Phys. Rev. Lett. 74, 1831 (1995).

${ }^{20}$ J. Rotvig, A.-P. Jauho, and H. Smith, Phys. Rev. B 54, 17691 (1996).

${ }^{21}$ K. I. Hino, K. Yashima, and N. Toshima, Phys. Rev. B 71, 115325 (2005).

${ }^{22}$ A. P. Jauho and K. Johnsen, Phys. Rev. Lett. 76, 4576 (1996).

${ }^{23}$ T. Meier, G. von Plessen, P. Thomas, and S. W. Koch, Phys. Rev. B 51, 14490 (1995).

${ }^{24}$ J. M. Lachaine, M. Hawton, J. E. Sipe, and M. M. Dignam, Phys. Rev. B 62, R4829 (2000).

${ }^{25}$ K. Unterrainer, B. J. Keay, M. C. Wanke, S. J. Allen, D. Leonard, G. Medeiros-Ribeiro, U. Bhattacharya, and M. J. W. Rodwell, Phys. Rev. Lett. 76, 2973 (1996).

${ }^{26}$ M. Glück, A. R. Kolovsky, and H. J. Korsch, Phys. Rep. 366, 103 (2002).

${ }^{27}$ A. H. Chin, J. M. Bakker, and J. Kono, Phys. Rev. Lett. 85, 3293 (2000).
} 
${ }^{28}$ A. Srivastava, R. Srivastava, J. Wang, and J. Kono, Phys. Rev. Lett. 93, 157401 (2004).

${ }^{29}$ J. Karczmarek, M. Stott, and M. Ivanov, Phys. Rev. A 60, R4225 (1999).

${ }^{30}$ Z.-G. Wang, D. Suqing, and X.-G. Zhao, Phys. Rev. B 69, 035305 (2004).

${ }^{31}$ H.-F. Liu and X.-G. Zhao, Int. J. Mod. Phys. B 14, 41 (2000).

${ }^{32}$ M. J. Zhu, X.-G. Zhao, and Q. Niu, J. Phys.: Condens. Matter 11, 4527 (1999).

${ }^{33}$ M. M. Dignam and C. M. de Sterke, Phys. Rev. Lett. 88, 046806 (2002).

${ }^{34}$ P. Domachuk, C. M. de Sterke, J. Wan, and M. M. Dignam, Phys. Rev. B 66, 165313 (2002).

${ }^{35}$ K. Hino and N. Toshima, Solid State Commun. 138, 341 (2006).

${ }^{36}$ T. W. Hänsch, Opt. Commun. 80, 71 (1990).

${ }^{37}$ Y. Kobayashi, Z. Y. Wei, M. Kakehata, H. Takada, and K. Torizuka, IEEE J. Sel. Top. Quantum Electron. 9, 1011 (2003).

${ }^{38}$ Y. Kobayashi, H. Takada, M. Kakehata, and K. Torizuka, Appl. Phys. Lett. 83, 839 (2003).

${ }^{39}$ D. D. Yavuz, D. R. Walker, M. Y. Shverdin, G. Y. Yin, and S. E. Harris, Phys. Rev. Lett. 91, 233602 (2003).

${ }^{40}$ M. Y. Shverdin, D. R. Walker, D. D. Yavuz, G. Y. Yin, and S. E. Harris, Phys. Rev. Lett. 94, 033904 (2005).

${ }^{41}$ M. Katsuragawa, K. Yokoyama, and T. Onose, Opt. Express 13, 5628 (2005).

${ }^{42} \mathrm{H}$. Haug and S. W. Koch, Quantum Theory of the Optical and Electronic Properties of Semiconductors, 3rd ed. (World Scientific, Singapore, 1994), Chap. 12.

${ }^{43}$ T. Meier, H. J. Kolbe, A. Thranhardt, G. Weiser, and S. W. Koch, Physica E (Amsterdam) 7, 267 (2000).

${ }^{44}$ M. M. Dignam, Phys. Rev. B 59, 5770 (1999).

${ }^{45}$ K. I. Hino, K. Goto, and N. Toshima, Phys. Rev. B 69, 035322
(2004).

${ }^{46}$ Indeed, the coefficient $a_{\nu \mathbf{K}}$ given in Eq. (18) is obtained straightforward from Eq. (9) in the $\lambda$ representation without the bypass into Eq. (11) in the $z$ representation. In this case, similar to Eq. (16), $p_{\lambda \lambda^{\prime} \mathbf{K}_{\|}}(t)$ is expanded in terms of the basis set $\left\{\psi_{\lambda \lambda^{\prime} \nu}(t)\right\}$, defined in Eq. (23). However, $\psi_{\lambda_{\lambda^{\prime} \nu}}(t)$ is generally less familiar than $\psi_{\nu}\left(z, z^{\prime}, t\right)$, and it seems unclear at first sight of Eq. (9) to see which form of the homogeneous equation, corresponding to Eq. (15), is satisfied by $\psi_{\lambda \lambda^{\prime} \nu}(t)$.

${ }^{47}$ W. V. Houston, Phys. Rev. 57, 184 (1940).

${ }^{48}$ The same notation of $z$ as that for the electronic coordinate defined right above Eq. (10) is used just within Sec. II C since it is not likely to cause unnecessary notational confusing.

${ }^{49}$ K. J. Hino and N. Toshima, Phys. Rev. B 71, 205326 (2005).

${ }^{50}$ S. Yoshida, C. O. Reinhold, and J. Burgdörfer, Phys. Rev. Lett. 84, 2602 (2000).

${ }^{51}$ N. Linder, Phys. Rev. B 55, 13664 (1997).

${ }^{52}$ It is seen that the arrow depicted in panel (f) of Fig. 3 indicates the relatively broad portion of the quasienergy band. In fact, this portion is composed of two bands overlapping each other. One arises from the photon sideband of the Floquet state $[B$ $=(2,2), J=-3$ ] forming the wider band, and the other arises from the parent band of the Floquet state $[B=(1,1), J=0]$ forming the narrower band. The former contributes little to the associated spectral portion (arrower in blue) in Fig. 7 due to no effect of the ac-ZT. Therefore, this portion of the spectra is actually ascribable to this parent band with the narrower quasienergy band.

${ }^{53} \mathrm{H}$. Römer, Theoretical Optics: An Introduction (Wiley-VCH, New York, 2005), Chap. 2.

${ }^{54}$ Z. Ficek and S. Swain, Quantum Interference and Coherence: Theory and Experiments (Springer, New York, 2005), Chap. 5. 